\title{
Asymptotic analysis of pore crack initiation near a free edge
}

\author{
Aurelien Doitrand ${ }^{\mathrm{a}, *}$, Dominique Leguillon ${ }^{\mathrm{b}}$ \\ ${ }^{a}$ Univ Lyon, INSA-Lyon, UCBL, CNRS, MATEIS, UMR5510, F-69621 Villeurbanne, France \\ ${ }^{b}$ IJLRA, Sorbonne Université, CNRS UMR 7190, Paris, France
}

\begin{abstract}
The matched asymptotic (MA) approach of the coupled criterion (CC) enables studying crack initiation at a pore located near a free edge, which leads to a competition between three possible crack initiation mechanisms, namely crack initiation in the ligament, at the pore side opposite to the free edge or simultaneously at both pore sides. The mechanism that is the most likely to occur depends on both the pore-to-free edge ligament size and the material characteristic length to pore diameter ratio. Simultaneous crack initiations at both pore sides occur for large enough ligament size. For smaller ligament sizes, unilateral pore crack initiation is more likely to occur, within the ligament for small characteristic length to pore diameter ratios and at the pore side opposite to the free edge otherwise. The CC MA approach enables estimating the initiation remote imposed stress as well as the initiation crack length.

Keywords: Coupled criterion; pore; matched asymptotic expansions; free edge
\end{abstract}

\section{Introduction}

Finite Fracture Mechanics (FFM) framework [1, 2, 3] consists in studying finite crack increments instead of infinitesimal ones as in classical Linear Elastic Fracture Mechanics (LEFM) approach [4]. Whereas LEFM assesses the propagation of an already existing crack, a main interest of FFM is the possibility to study its nucleation. Among this framework, the coupled criterion (CC) [5] has proved to be an efficient approach for crack initiation assessment in various situations [6]. This approach is based on the simultaneous fulfillment of a stress condition and of a discrete energy balance between the states prior and after crack initiation. It requires as inputs the material strength and critical energy release rate and enables the determination of the initiation loading and corresponding crack increment.

\footnotetext{
*Corresponding author

Email address: aurelien.doitrand@insa-lyon.fr (Aurelien Doitrand)
} 
The CC application is based on the calculation of the stress field prior to crack initiation and of the potential energy release as a function of the crack length. These functions can be obtained through existing available analytical functions [7] or more generally calculated by means of Finite Element (FE) simulations of the problem under investigation. The CC is computationally efficient since it generally requires only a few linear elastic calculations. It can also account for nonlinearities [8, 9, 10, 11]. The CC efficiency compared to other fracture modeling approach is particularly highlighted in the context of inverse identification approaches of the fracture parameters [12, 13]. Indeed, contrary to other modeling approaches such as cohesive zone models, the material strength and critical energy release rate are only implemented during the post-processing phase of the FE calculations.

The original implementation of the CC was established by Leguillon [5] to study crack initiation at $\mathrm{V}$-notches based on the matched asymptotic (MA) expansion procedure developed by Leguillon and Sanchez-Palencia [14]. The MA approach focuses on a domain surrounding the initiation crack zone. The boundary conditions imposed to this domain are asymptotic displacement or stress fields corresponding to the studied geometry (such as, e.g., a V-notch, a hole or a bimaterial interface). It relies on the assumptions that the initiation crack increment is small and far enough from the structure boundaries that may influence these displacement and stress fields. Under these assumptions, the full-FE and MA approaches yields equivalent results [15, 16, 17, 18, 19], the MA approach being even more computationally efficient since it requires fewer calculations without modeling the whole specimen geometry.

Crack initiation at a circular hole using the CC was studied by several authors, mainly based on analytical or full-FE approaches. Weißgraeber et al. [6] set up a closed form analytical CC formulation to assess crack initiation at elliptical holes in an infinite medium under remote tensile loading and studied the aspect ratio and size influence on the imposed stress at initiation. The elliptical hole problem was addressed using the CC by Carpinteri et al. [20]. Sapora and Cornetti [21] studied crack initiation at a circular hole in infinite medium under biaxial loadings and the stability of the initiated crack depending on the loading biaxiality. Asymmetric crack initiation in a plate with a centered hole under combined tension bending was studied by Rosendahl et al. [22]. They highlighted a competition between three mechanisms, namely asymmetric crack initiation at the hole, crack initiation at only one hole side or at the specimen free edge, that may occur depending on the hole size and bending to tensile stress ratio. The $\mathrm{CC}$ was also implemented to assess crack initiation in PMMA specimens containing a circular hole under tensile loading and 
enabled retrieving the failure stress variation from the material tensile strength for small holes to one third of it for large enough holes [23, 24, 25]. Romani et al. [26] tested gypsum specimens with a circular hole under compression and detected crack initiation using digital image correlation, the initiation stress predictions were in agreement with the experimental measurements. The CC was also successfully used to predict the initiation stress in Brazilian Disk tests on polymer specimen with a centered hole, taking into account the nonlinear material behavior [1].

The CC was also applied to predict crack initiation in composite specimens with a hole . Martin et al. [27, 28, enriched the classical point stress criterion by considering both stress and energy conditions. They highlighted the limit of the point stress criterion by evidencing that the initiation crack length depends on the hole size to specimen width ratio. Camanho et al. [29] studied a similar problem based on analytical formulae of the stress and energy conditions for different laminate layups and highlighted the suitability of the approach to predict the initiation stress for configurations not exhibiting debonding as main damage mechanism, which would have required extra FE calculations to implement the CC. Catalanotti et al. [30] predicted net tension crack initiation in fastened joints composites by modeling the fastener influence on the hole as a contact pressure on the hole contour. Felger et al. 31] studied the influence of the composite orientation with respect to tensile direction on mixed mode crack initiation in composites specimens with a hole and correctly covered the experimentally observed size effect and the influence of the material anisotropy on the plate's effective strength. This was done employing the matched asymptotic approach of the CC [32], which was also used by Leguillon et al. [33] to examine the problem of a crack blunted by a pore and the influence of two neighboring pores ahead of a primary crack.

The abovementioned works refer to crack initiation at a circular hole either in infinite medium or centered in a specimen of finite width. However, few works consider the interaction between a pore and a free edge [34]. The objective of this work is to implement the MA approach of the CC to the problem of crack initiation around a pore close to a free edge. The CC MA approach is explained in Section 2 and an example of CC solution is detailed in Section 3 . The conditions for crack initiation within the pore-to-free edge ligament(either from the pore or from the free edge) are described in Section 4 and other crack initiation mechanisms such as crack initiation beyond the pore or simultaneous crack initiation at both pore sides are finally studied in Section 5 . 


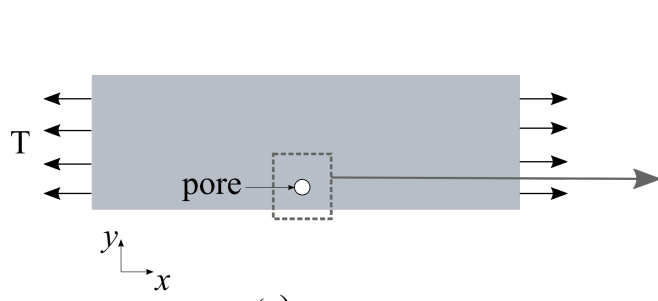

(a)

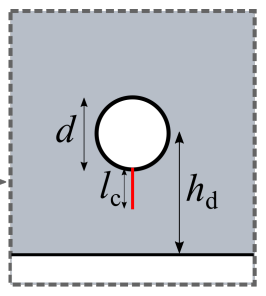

(b)

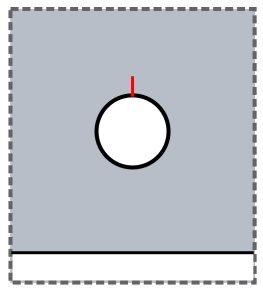

(c)

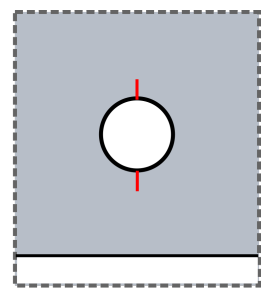

(d)

Figure 1: a) Specimen containing a pore close to a free edge under tensile loading. Crack initiation scenarii at the hole b) in the pore-to-free edge ligament, c) at the pore side opposite to the free edge or d) at both pore sides.

\section{Crack initiation at a pore near a free edge}

\subsection{Matched asymptotic approach}

We consider the problem of crack initiation in the vicinity of a pore that is close to a free edge, under tensile loading for instance as depicted in Fig. 13. The stress concentrations induced around the pore may lead to several scenarii: a crack initiating at the pore side in the pore-to-free edge ligament, a crack initiating at the pore side opposite to the free edge or simultaneous crack initiation at both pore sides. This two dimensional model is representative of either a cylindrical cavity (extrusion along the third direction) or a spherical cavity (in the cavity middle plane).

The MA approach consists in studying two configurations, the first one at the scale of the specimen, in which the pore is small compared to the specimen dimensions and can thus be neglected, and the second one focusing only on a zone close to the pore. The matching of both configurations has to be made in an intermediate zone in order to derive expressions for the stress and the potential energy from which crack initiation will be determined using the CC. We thus consider a two-scale problem to solve under plane strain linear elasticity assumption. The first problem is written at the specimen scale. The displacement field $\boldsymbol{U}^{d}$ (the superscript $d$ refers to the presence of a pore of diameter $d$ ) is solution of the following set of equations:

$$
\left\{\begin{array}{l}
-\nabla \cdot \underline{\sigma}\left(\boldsymbol{U}^{d}\right)=0 \\
\underline{\sigma}\left(\boldsymbol{U}^{d}\right)=\underline{\underline{C}}: \nabla \boldsymbol{U}^{d} \\
\underline{\sigma}\left(\boldsymbol{U}^{d}\right) \cdot \boldsymbol{n}=\boldsymbol{O} \text { along the free edge, } \boldsymbol{n} \text { is the normal to the free edge. }
\end{array}\right.
$$

It is assumed that the pore diameter $d$ and the crack initiation length $\left(\ell_{c}\right)$ at the pore are relatively small compared to the specimen dimensions, this assumption has to be checked afterwards to ensure 
the validity of the MA approach. Therefore, the actual solution can be approximated by:

$$
\boldsymbol{U}^{d}\left(x_{1}, x_{2}, l\right)=\boldsymbol{U}^{0}\left(x_{1}, x_{2}, 0\right)+\text { small correction }
$$

where $\boldsymbol{U}^{0}\left(x_{1}, x_{2}, 0\right)$ is the solution to an idealized problem without pore, e.g. tensile loading of a plain specimen $(d=0)$. The correction in Eq. (2) decreases to 0 when $d$ tends towards 0 . This approximation is valid except near the pore, it is called the outer field. The asymptotic displacement field corresponding to a straight free edge can be calculated as $\operatorname{Tr}^{\lambda} \boldsymbol{t}(\theta)+o\left(r^{\lambda}\right)$, where $\lambda=1$ and $\boldsymbol{t}(\theta)$ are obtained solving an eigenvalue problem [14]. $T$ represents the Generalized Stress Intensity Factor (GSIF) for a free edge, i.e. the remote imposed stress for a pure tensile loading. The displacement field in the plain specimen can thus be written as:

$$
\boldsymbol{U}^{0}\left(x_{1}, x_{2}, 0\right)=\boldsymbol{U}^{0}(0,0)+\operatorname{Tr} \boldsymbol{t}(\theta)+\ldots
$$

To have a detailed form of the actual solution $\boldsymbol{U}^{d}$, the initial domain is stretched by $1 / d$. We define the new dimensionless space variables as $y_{i}=x_{i} / d$ and the dimensionless crack length $\mu=\ell / d$. The dimensionless distance between the free edge and the pore tip is $\varepsilon=\left(h_{d}-d / 2\right) / d$. As d tends towards 0 , the corresponding domain becomes unbounded. It is called the inner domain, in which the pore diameter is 1 . The inner domains in the three possible initiation configurations (initiation in the pore-to-free edge ligament, on the pore side opposite to the free edge or simultaneously on both pore sides) are depicted in Fig. 2. The actual solution is assumed to expand in the following way:

$$
\boldsymbol{U}^{d}\left(x_{1}, x_{2}, \ell\right)=\boldsymbol{U}^{d}\left(d y_{1}, d y_{2}, d \mu\right)=F_{0}(d) \boldsymbol{V}^{0}\left(y_{1}, y_{2}, \mu\right)+F_{1}(d) \boldsymbol{V}^{1}\left(y_{1}, y_{2}, \mu\right)
$$

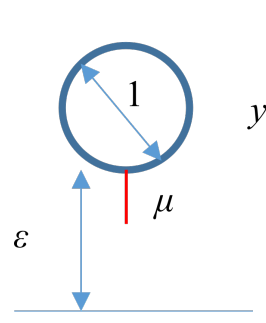

(a)

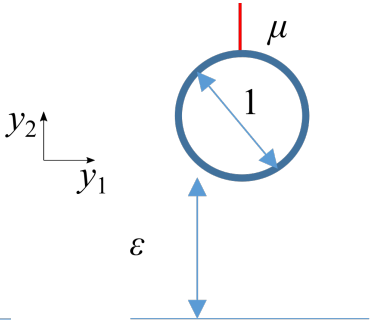

(b)

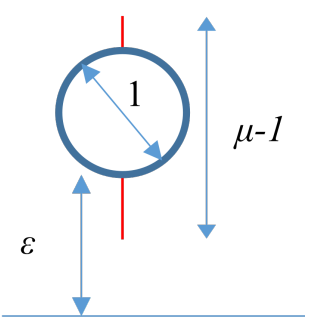

(c)

Figure 2: Inner domain sketch for crack initiation a) in the pore-to-free edge ligament, b) at the pore side opposite to the free edge or c) at both pore sides. 
with

$$
\lim _{d \rightarrow 0} \frac{F_{1}(d)}{F_{0}(d)}=0
$$

The $\boldsymbol{V}^{i}$ form the inner field, they are solutions to problems with prescribed behaviour at infinity. They must match at infinity with the behaviour of the far field. Therefore there exists an area, far from the free edge in the inner expansion and close to it in the outer expansion where both expansions given in Eq. (2) and Eq. (4) hold true. Matching the terms in Eq. (3) and Eq. (4) leads to :

$$
\left\{\begin{array}{l}
F_{0}(d)=1 \\
\boldsymbol{V}^{0}\left(y_{1}, y_{2}, \mu\right)=\boldsymbol{U}^{0}(0,0) \\
F_{1}(d)=T d \\
\boldsymbol{V}^{1}\left(y_{1}, y_{2}, \mu\right) \approx \gamma \boldsymbol{t}(\theta)
\end{array}\right.
$$

with $\gamma=r / d$. The symbol $\approx$ means that $\boldsymbol{V}^{1}$ behaves like $\gamma \boldsymbol{t}(\theta)$ at infinity, it can thus be written as:

$$
\boldsymbol{V}^{1}\left(y_{1}, y_{2}, \mu\right)=\gamma \boldsymbol{t}(\theta)+\hat{\boldsymbol{V}}^{1}\left(y_{1}, y_{2}, \mu\right)
$$

We have to prove that $\hat{\boldsymbol{V}}^{1}\left(y_{1}, y_{2}, \mu\right)$ exists and verifies the equilibrium equations. By combining Eqs. (4) and (6) into Eq. (1), and noticing that the free edge remains a free edge in the inner domain, $\hat{\boldsymbol{V}}^{1}$ is solution to the following problem:

$$
\left\{\begin{array}{l}
-\nabla_{y} \cdot \underline{\hat{\sigma}}=0 \text { where } \nabla_{y}=\frac{1}{d} \nabla_{x} \\
\underline{\hat{\sigma}}=\underline{\underline{C}}: \nabla_{y} \hat{\boldsymbol{V}}^{1} \\
\underline{\hat{\sigma}} \cdot \boldsymbol{n}_{1}=-T \cdot \boldsymbol{n}_{2} \text { along the free edge, } \boldsymbol{n}_{1}=(0,1), \boldsymbol{n}_{2}=(1,0) \\
\underline{\hat{\sigma}} \cdot \boldsymbol{n}_{2}=-T \cdot \boldsymbol{n}_{2} \text { along the crack faces and the pore edge } \\
\underline{\hat{\sigma}} \cdot \boldsymbol{n}=-T \cdot \boldsymbol{n}_{2} \text { along the pore edge } \\
\hat{\boldsymbol{V}}^{1} \text { decreases at infinity }
\end{array}\right.
$$

The system of equations has a unique solution with a finite energy [14] (extension of Lax-Milgram theorem to unbounded domains). As a consequence of the finite energy, the solution decreases to 0 at infinity. The expansion finally writes:

$$
\boldsymbol{U}^{d}\left(x_{1}, x_{2}, \ell\right)=\boldsymbol{U}^{d}\left(d y_{1}, d y_{2}, d \mu\right)=\boldsymbol{U}^{0}(0,0)+T d\left[\gamma \boldsymbol{t}(\theta)+\hat{\boldsymbol{V}}^{1}\left(y_{1}, y_{2}, \mu\right)\right]
$$


The change in potential energy is written as [14, 35]

$$
\begin{aligned}
-\delta W_{p} & =\Psi\left(\boldsymbol{U}^{d}\left(x_{1}, x_{2}, \ell\right), \boldsymbol{U}^{d}\left(x_{1}, x_{2}, 0\right)\right) \\
& =\Psi\left(\boldsymbol{U}^{d}\left(x_{1}, x_{2}, \ell\right), \boldsymbol{U}^{0}\left(x_{1}, x_{2}\right)\right)-\Psi\left(\boldsymbol{U}^{d}\left(x_{1}, x_{2}, 0\right), \boldsymbol{U}^{0}\left(x_{1}, x_{2}\right)\right)
\end{aligned}
$$

where

$$
\Psi(\boldsymbol{f}, \boldsymbol{g})=\frac{1}{2} \int_{\Gamma}[\underline{\sigma}(f) \cdot \boldsymbol{n} \cdot \boldsymbol{g}-\underline{\sigma}(g) \cdot \boldsymbol{n} \cdot \boldsymbol{f}] d s
$$

$\Psi$ is a path independent integral where $\Gamma$ is any closed contour starting and finishing on the free edge and surrounding the studied crack initiation location, $\boldsymbol{n}$ is the inwards normal to this contour. Thus, it comes:

$$
\begin{aligned}
-\delta W_{p} & =T^{2} d^{2} \Psi\left(\gamma \boldsymbol{t}(\theta), \boldsymbol{V}^{1}\left(y_{1}, y_{2}, 0\right)\right)+T^{2} d^{2} \Psi\left(\boldsymbol{V}^{1}\left(y_{1}, y_{2}, \mu\right), \gamma \boldsymbol{t}(\theta)\right) \\
& =T^{2} d^{2}\left[\Psi\left(\boldsymbol{V}^{1}\left(y_{1}, y_{2}, \mu\right), \gamma \boldsymbol{t}(\theta)\right)-\Psi\left(\boldsymbol{V}^{1}\left(y_{1}, y_{2}, 0\right), \gamma \boldsymbol{t}(\theta)\right)\right] \\
& =T^{2} d^{2}[B(\mu)-B(0)]
\end{aligned}
$$

with $B(\mu)=\Psi\left(\boldsymbol{V}^{1}\left(y_{1}, y_{2}, \mu\right), \gamma \boldsymbol{t}(\theta)\right)$. According to the expansion before crack initiation, the stress field writes:

$$
\underline{\sigma}(\mu)=T \underline{\tilde{\sigma}}_{\varepsilon}(\mu)
$$

The stress function $\tilde{\sigma}_{\varepsilon}$ can be computed in the inner domain bounded at a large distance for a given dimensionless distance between the pore tip and the free edge $\varepsilon$. The index $\varepsilon$ in $\underline{\tilde{\sigma}}$ indicates that this function is computed for a given value of $\varepsilon$, the MA approach thus enables the calculation of the stress variation along the crack path prior to crack initiation as well as the potential energy release as a function of the dimensionless crack length. These two functions are used in the sequel to implement the coupled criterion to assess crack initiation.

\subsection{The coupled criterion}

The coupled criterion states that crack initiation can occur if two conditions are fulfilled [5]:

- the stress must be larger than the material strength $\sigma_{c}$ along the crack path before initiation,

- the potential energy released by crack opening must be larger than the energy required for crack nucleation: $\mathcal{G}_{c} \ell$, where $\mathcal{G}_{c}$ is the material critical energy release rate. 
The second condition leads to defining the incremental energy release rate $G_{i n c}=-\delta W_{p} / \ell=$ $-\delta W_{p} /(d \mu)$, which can be calculated using Eq. (13):

$$
G_{i n c}(\mu)=T^{2} d \frac{B(\mu)-B(0)}{\mu}=T^{2} d g_{\varepsilon}(\mu) \frac{1-\nu^{2}}{E}
$$

The dimensionless function $g_{\varepsilon}(\mu)=\frac{B(\mu)-B(0)}{\mu} \frac{E}{1-\nu^{2}}$ can be obtained by computing the potential energy difference as a function of the dimensionless crack length in the inner domain. It is done by finite element calculations, the inner domain being artificially bounded at a distance large with respect to $\mu, \varepsilon$ and 1 (the dimensionless pore size in the inner domain). The index $\varepsilon$ in $g_{\varepsilon}$ indicates that this function is computed for a given value of $\varepsilon$, for several dimensionless crack lengths $\mu$. It can be checked that the function $g_{\varepsilon}(\mu)$ is independent of the material Young's modulus and Poisson's ratio. Applying the coupled criterion $G_{i n c}(\mu) \geq \mathcal{G}_{c}$ and $\sigma(\mu) \geq \sigma_{c}$ all along the crack path prior to initiation, it results in the following equation system to be solved:

$$
\left\{\begin{array}{l}
T^{2} d g_{\varepsilon}(\mu) \frac{1-\nu^{2}}{E} \geq \mathcal{G}_{c} \\
T \tilde{\sigma}_{\varepsilon}(\mu) \geq \sigma_{c}
\end{array}\right.
$$

Combining both equations finally yields the equation that must be solved to determine the dimensionless initiation crack length $\mu_{c}$ and the corresponding initiation crack length $\ell_{c}=d \mu_{c}$ :

$$
\frac{g_{\varepsilon}(\mu)}{\tilde{\sigma}_{\varepsilon}(\mu)^{2}} \frac{1-\nu^{2}}{E}=\frac{1}{d} \frac{\mathcal{G}_{c}}{\sigma_{c}^{2}}
$$

which rewrites as:

$$
\frac{g_{\varepsilon}(\mu)}{\tilde{\sigma}_{\varepsilon}(\mu)^{2}}=\frac{1}{d} \frac{E \mathcal{G}_{c}}{\left(1-\nu^{2}\right) \sigma_{c}^{2}}=\frac{\ell_{m a t}}{d}
$$

where $\ell_{m a t}$ is the material characteristic length defined as $\ell_{m a t}=\frac{E \mathcal{G}_{c}}{\left(1-\nu^{2}\right) \sigma_{c}^{2}}$. Eq. 18 can be solved using Newton's method for instance. Once Eq. [18] is solved to determine the dimensionless initiation crack length $\mu_{c}$, the critical GSIF at initiation is obtained as:

$$
T_{c}=\sqrt{\frac{E \mathcal{G}_{c}}{\left(1-\nu^{2}\right) d g_{\varepsilon}\left(\mu_{c}\right)}}=\sigma_{c} \sqrt{\frac{\ell_{m a t}}{d} \frac{1}{g_{\varepsilon}\left(\mu_{c}\right)}}=\frac{\sigma_{c}}{\tilde{\sigma}_{\varepsilon}\left(\mu_{c}\right)}
$$

The CC implementation thus enables the determination of both the initiation crack length and remote imposed stress. It can be noted that the remote failure stress depends on the pore size since the actual pore diameter $d$ appears in Eqs. 18$)$ and $(19 p)$. 


\subsection{Finite Element calculations}

For a given dimensionless pore to free edge ligament size $\varepsilon$, two functions are computed by means of FE calculations, namely the dimensionless stress $\tilde{\sigma}_{\varepsilon}$ and incremental energy release rate $g_{\varepsilon}$. Both functions are computed in the inner domain for several dimensionless crack lengths $\mu$. The inner domain is theoretically unbounded so that in FE calculations, a domain sufficiently large with respect to the small parameters, i.e. the pore size which is equal to 1 and the dimensionless ligament size and crack length. It can be noted that the validity of the MA approach also relies on the fact that the small parameters all are in the same order of magnitude, therefore the calculation are limited to dimensionless crack lengths $0<\mu<10$. A square with side $L$ inner domain is defined, and boundary conditions consist in prescribed displacements (Eq. (3p) at the domain fictitious boundaries. These displacement writes:

$$
\left\{\begin{array}{l}
\boldsymbol{U}_{x}^{0}\left(y_{1}, y_{2}\right)=\frac{1-\nu^{2}}{E} T y_{1} \\
\boldsymbol{U}_{y}^{0}\left(y_{1}, y_{2}\right)=\frac{-\nu(1+\nu)}{E} T y_{2}
\end{array}\right.
$$

Since the inner domain is theoretically unbounded, the fictitiously bounded domain size used for the FE calculations must be large enough so that it has no influence on the calculation of $\tilde{\sigma}_{\varepsilon}$ and $g_{\varepsilon}$. We performed calculations for inner domain sizes $L=100,200,500,1000$ and 5000. Fullintegration plain strain four-node elements are used. The meshes are refined in the vicinity of the pore, the mesh size is set to $\min (1 / 10, \varepsilon / 10)$ in the pore to free edge ligament and $1 / 10$ along the crack path on the pore side opposite to the free edge. Whatever the inner domain size used for FE calculations, differences on dimensionless stress along the crack path before initiation smaller than $0.1 \%$ are obtained. Fig. 3 shows the dimensionless incremental energy release rate and the ratio involve in Eq. (18) variations as a function of the dimensionless crack length for several inner domain sizes. Whatever the inner domain size, almost no differences are observed on the pore to free edge ligament. For a $L=100$ domain size, differences between $1 \%$ and $10 \%$ are obtained compared to the case with a $L=5000$ inner domain size, for dimensionless crack lengths $\mu>2$. These differences reduce to between $1 \%$ and $3 \%$ for $\mu>5$ for $L=200$ and down to $0.3 \%$ for $L=500$. For $L=1000$, differences smaller than $0.1 \%$ are obtained. In practice, the size of the domain can thus be set to $100 \max (\mu, \varepsilon)=1000$, which results in FE meshes containing around 3000 nodes. Since the crack lies on a symmetry plane of the inner domain, only one half is modeled and the crack length is increased to compute $g_{\varepsilon}$ by successively releasing the symmetry condition on the crack path nodes. For a given crack initiation configuration, $10 \mathrm{FE}$ calculations with varying $\varepsilon$ 
are performed, each calculation lasts around 1 minute. If the actual pore-to-free edge ligament size does not strictly correspond to a calculated $\varepsilon$ value, crack initiation length and stress are obtained by interpolation of the computed values.

\section{Example of CC solution}

We illustrate the matched asymptotic approach CC solution with the following example of a pore which diameter is $0.1 \mathrm{~mm}$, the pore center is located at $0.15 \mathrm{~mm}$ from the free edge loaded in tension in a material with the following properties: $E=3000 \mathrm{MPa}, \nu=0.2, \sigma_{c}=10 \mathrm{MPa}$, $\mathcal{G}_{c}=5 \mathrm{~J} / \mathrm{m}^{2}$. The corresponding characteristic length is $l_{\text {mat }}=0.156 \mathrm{~mm}$. The corresponding dimensionless distance to the porosity is $\varepsilon=\left(h_{d}-d / 2\right) / d=1$. The functions $g_{1}$ and $\tilde{\sigma}_{1}$ are computed as a function of the dimensionless distance $y_{2}$ or crack length $\mu=\ell / d$ (Fig. 4). In Fig. 4 b-c), $g_{1}$ and $\tilde{\sigma}_{1}$ are displayed as a function of the dimensionless crack length. In this first example, we only consider crack initiation in the pore to free edge ligament. Because of the stress concentrations at the pore side, the crack is likely to initiate from the pore ( $c f$. Fig. 2). Therefore, the dimensionless crack length $\mu=0$ corresponds to the pore side located towards the free edge, whereas $\mu=\varepsilon$ corresponds to a crack that breaks the ligament between the pore and the free edge and $\mu>1$ to a crack which reinitiates on the pore side opposite to the free edge. The functions $g_{\varepsilon}$, $\tilde{\sigma}_{\varepsilon}$ and thus $g_{\varepsilon} / \tilde{\sigma}_{\varepsilon}^{2}$ are neither continuous nor monotonous because of the pore, which has a direct implication in the CC solution. Indeed, solving Eq. (18) enables determining the dimensionless initiation crack length $\mu_{c}$. However, remember that the stress condition of the CC must be fulfilled

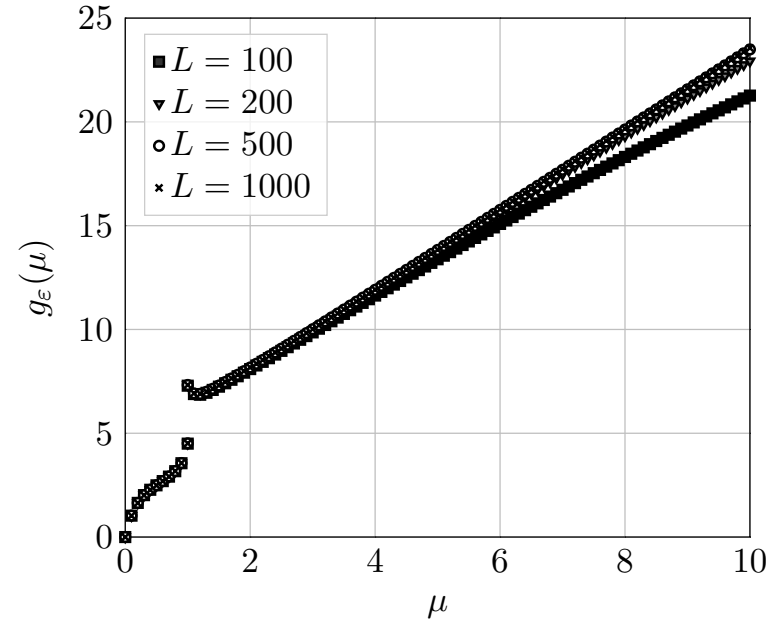

(a)

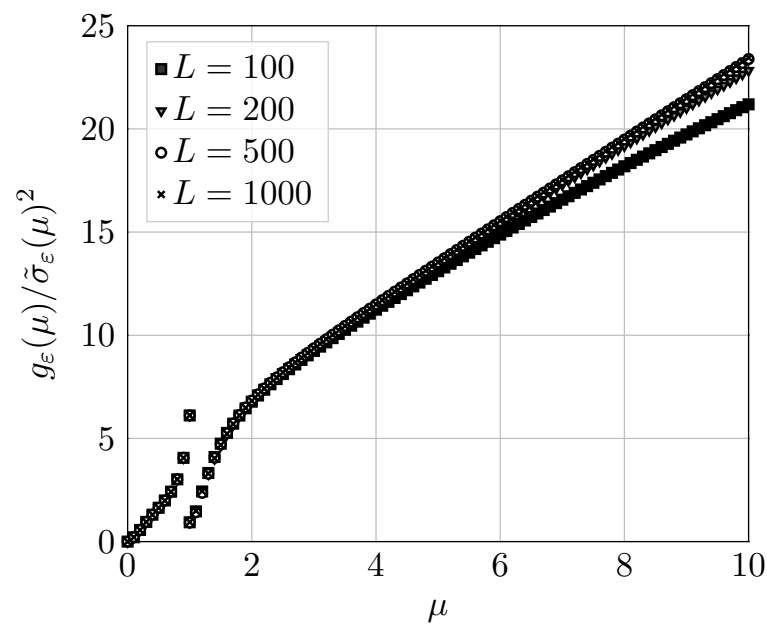

(b)

Figure 3: Dimensionless a) incremental energy release rate b) normalized by the square dimensionless stress as a function of the dimensionless crack length for $\varepsilon=1$. 
on the whole crack path just before initiation. This is directly checked if $\mu_{c}<\varepsilon$, i.e. the crack initiates in the ligament between the pore and the free edge since in this region, both $g_{\varepsilon}$ and $\tilde{\sigma}_{\varepsilon}$ are monotonous. If solving Eq. (18) yields a dimensionless initiation crack length $\mu_{c}>\varepsilon$, it has to be checked afterwards that the stress conditions is verified all along the crack path ( $c f$. Section 4 ).

Fig. 5 shows the variation of $g_{\varepsilon} / \tilde{\sigma}_{\varepsilon}^{2}$ as a function of $\mu$ as well as an illustration of Eq. (18) which solution enables the determination of the dimensionless initiation crack length $\mu_{c}=0.49<\varepsilon$. The initiation stress is determined using Eq. (19), which yields $T_{c}=8.0 \mathrm{MPa}$. The corresponding initiation crack length is $\ell_{c}=d \mu_{c}=0.049 \mathrm{~mm}$. Another way to solve the coupled criterion consists in rewriting Eqn. (16) so that:

$$
\left\{\begin{array}{l}
T \geq \sqrt{\frac{E \mathcal{G}_{c}}{\left(1-\nu^{2}\right) d g_{\varepsilon}(\mu)}}=\sigma_{c} \sqrt{\frac{\ell_{\operatorname{mat}}}{d g_{\varepsilon}(\mu)}} \\
T \geq \frac{\sigma_{c}}{\tilde{\sigma}_{\varepsilon}(\mu)}
\end{array}\right.
$$

which reverts to determining $\mu$ so that

$$
T \geq \sigma_{c} \min _{\mu} \max \left(\sqrt{\frac{\ell_{\text {mat }}}{d g_{\varepsilon}(\mu)}}, \frac{1}{\tilde{\sigma}_{\varepsilon}(\mu)}\right)
$$

Fig. 6a shows the variation of the remote stress required to fulfill either the stress or the energy criterion as a function of the dimensionless crack length. Solving Eqn. (22) reverts to first determine for a fixed $\mu$ the maximum stress required to fulfill both criteria and then to find its minimum $\mu_{c}$. This is equivalent to display $G_{i n c} / \mathcal{G}_{c}$ and $\sigma / \sigma_{c}$ and compare these functions to $1, \mu_{c}$ being determined as the length for which both criteria first exceed 1 for a minimum imposed loading.

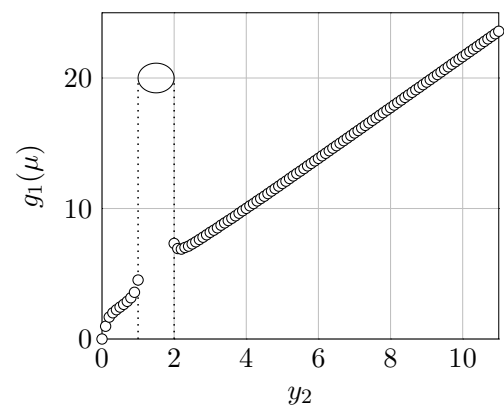

(a)

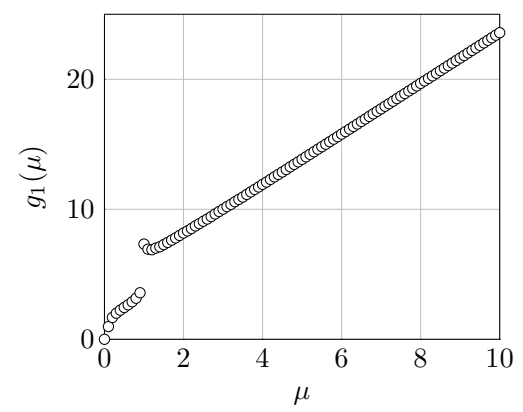

(b)

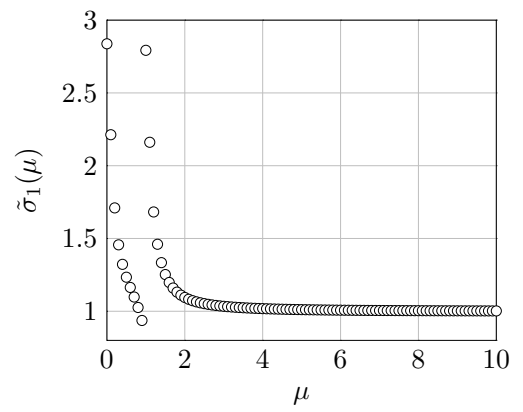

(c)

Figure 4: Dimensionless a-b) incremental energy release rate and c) stress as a function of a) the distance to the free edge $y_{2}$ or $\mathrm{b}-\mathrm{c}$ ) the dimensionless crack length $\mu$ for a dimensionless ligament size $\varepsilon=1$. The pore position is highlighted on a). 


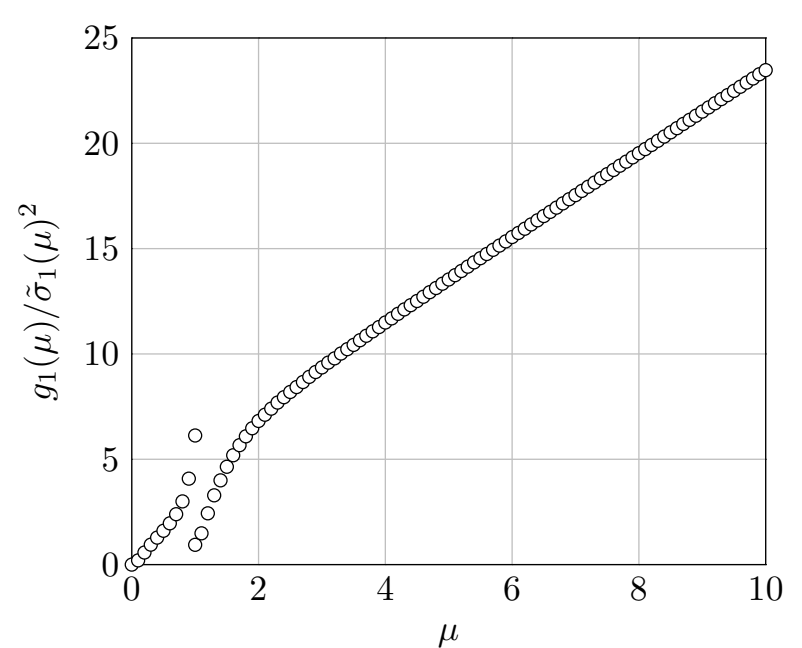

(a)

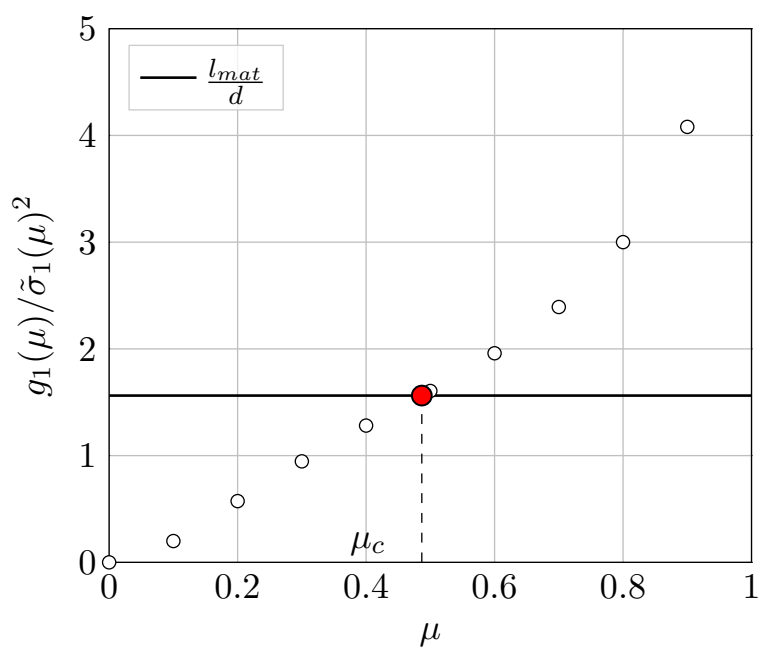

(b)

Figure 5: a) Dimensionless incremental energy release rate to square stress ratio as a function of the dimensionless crack length. b) Graphical representation of solution of Eqn. 18 enabling the initiation crack length determination.

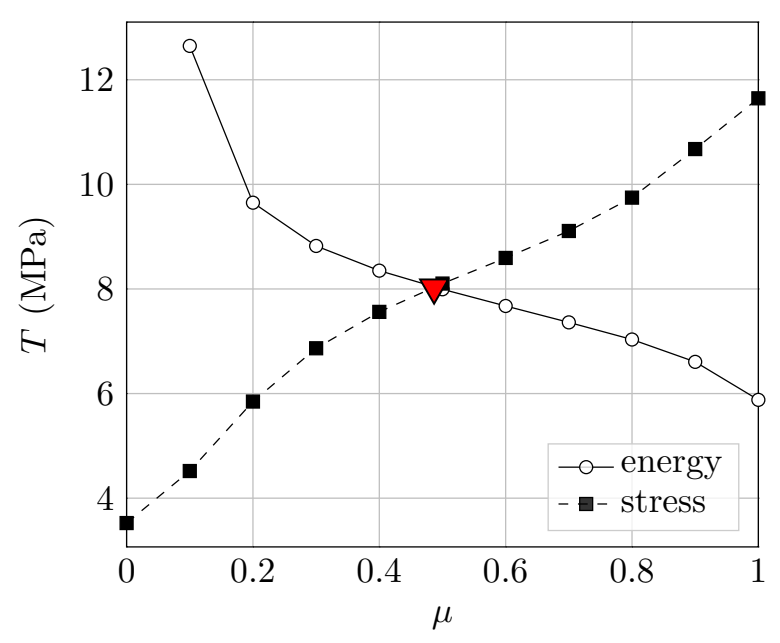

(a)

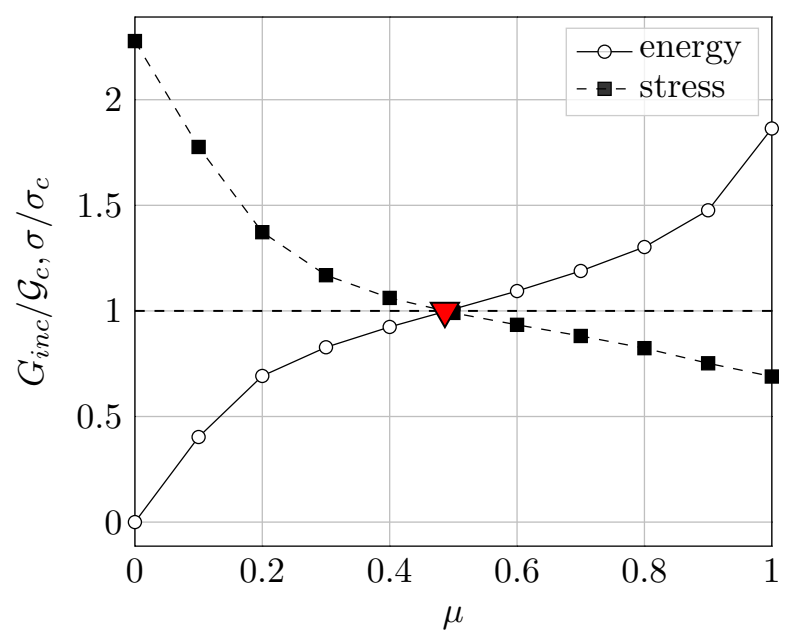

(b)

Figure 6: a) Imposed remote stress required to fulfill either the stress or the energy criterion and b) stress to strength and incremental to critical energy release rate ratio as a function of the crack length. The intersection of the two curves yields the initiation stress and crack length.

\section{Crack initiation in the pore to free edge ligament}

This section is dedicated to crack initiation occurring in the pore to free edge ligament. Other crack initiation mechanisms are considered in Section 5. We remind that the match asymptotic expansion approach remains valid provided $d$ is small compared to the dimensions of the specimen, and the small parameters $\mu_{c}$ and $\varepsilon$ are in the same order of magnitude as 1 (dimensionless pore diameter in the inner domain). Therefore, we perform calculations in the inner domain up to $\varepsilon=5$, meaning that the ligament size is at most five times the pore diameter. The functions $g_{\varepsilon}$ and $\tilde{\sigma}_{\varepsilon}$ 


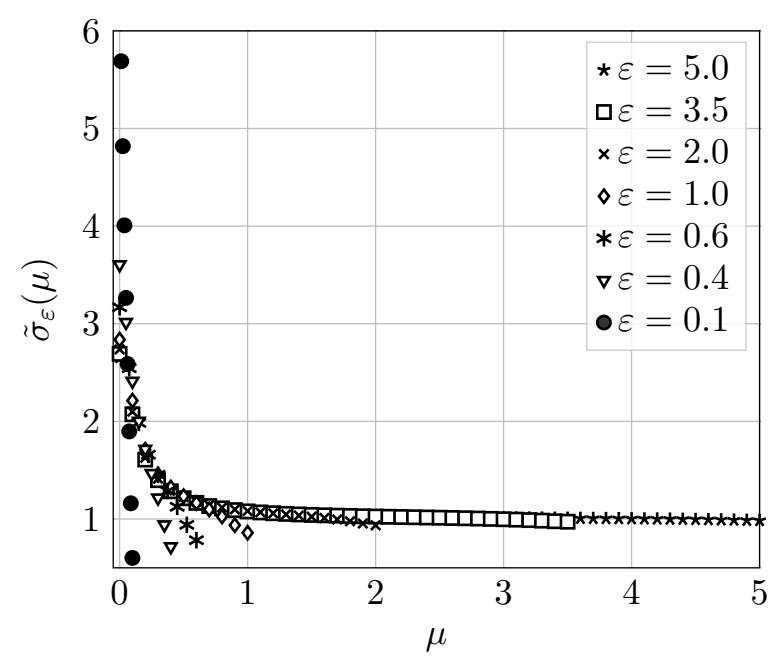

(a)

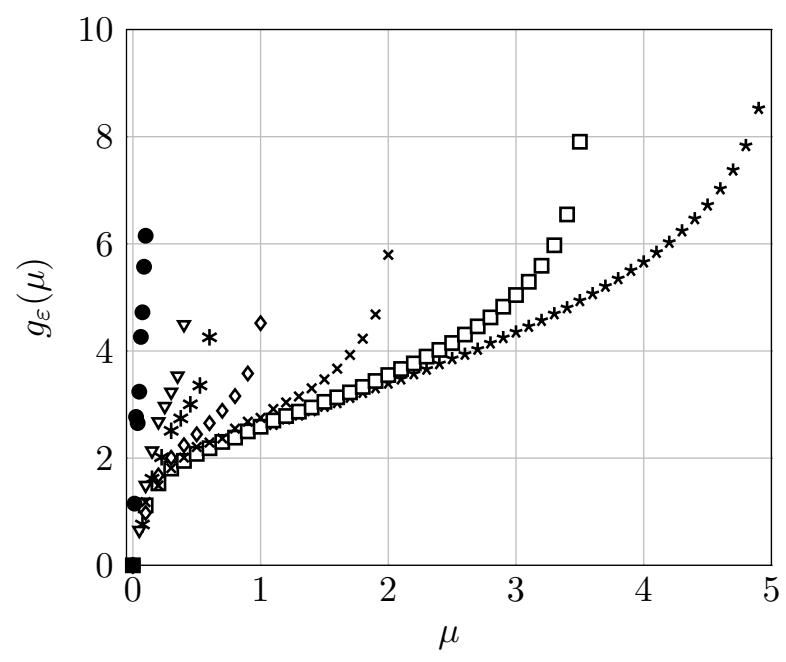

(b)

Figure 7: Dimensionless a) stress and b) incremental energy release rate as a function of the dimensionless crack length for several pore-to-free edge dimensionless ligament sizes.

are computed within the ligament for several dimensionless pore-to-free edge ligament sizes $\varepsilon$ (Fig. 7). One one hand, whatever the dimensionless ligament size, $g_{\varepsilon}$ increases monotonously over the ligament ((Fig. 7)b). On the other hand, $\tilde{\sigma}_{\varepsilon}(\mu)$ decreases monotonously on the ligament whatever the dimensionless ligament size ((Fig. 7)a). Besides, it decreases down to a value smaller than 1 , which will have an importance for initiation crack length of the same order of magnitude as the ligament size (see Section 4.2) or crack initiation lengths larger than the ligament size (see Section 5). The function $g_{\varepsilon}(\mu) / \tilde{\sigma}_{\varepsilon}(\mu)^{2}$ is depicted in Fig. 8 as well as the dimensionless initiation crack length $\mu_{c}$ depending on $\ell_{\text {mat }} / d$ for several dimensionless ligament sizes. Because of the monotonous variations of $g_{\varepsilon}$ and $\tilde{\sigma}_{\varepsilon}, g_{\varepsilon} / \tilde{\sigma}_{\varepsilon}$ is monotonously increasing (Fig. 8) thus Eqn. (18) has at most one solution over the ligament size provided $\ell_{\text {mat }} / d$ is smaller or equal to the maximum value of $g_{\varepsilon}(\mu) / \tilde{\sigma}_{\varepsilon}(\mu)$. This maximum value is the smallest for $\varepsilon=1$ and increases with increasing $\varepsilon>1$ or decreasing $\varepsilon<1$ (Fig. 8a). Fig. 8b shows the dimensionless crack length variations as a function of $\ell_{\text {mat }} / d$ for several $\varepsilon$. The dimensionless initiation crack length increases with increasing $\ell_{\text {mat }} / d$. For a fixed dimensionless ligament size, crack initiation in the ligament may occur only for a given range of $\ell_{\text {mat }} / d$ otherwise Eqn. (18) has no solution in the interval $0<\mu<\varepsilon$. It means that crack initiation in the ligament is constrained to a given range of $\ell_{\text {mat }} / d$ values, the case of larger $\ell_{\text {mat }} / d$ is studied in Section 5 . The range of $\ell_{\text {mat }} / d$ enabling crack initiation in the ligament is the smallest for $\varepsilon=1$ corresponding to $0 \leq \ell_{\text {mat }} / d \leq 6.1$. It means that if the ligament size is equal to the pore diameter, crack initiation will not occur in the ligament for materials having a characteristic length 


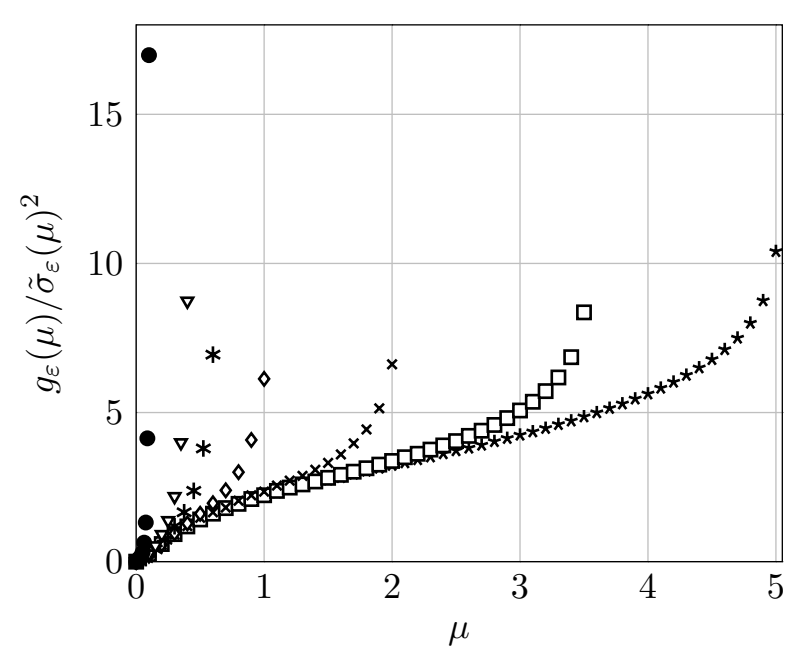

(a)

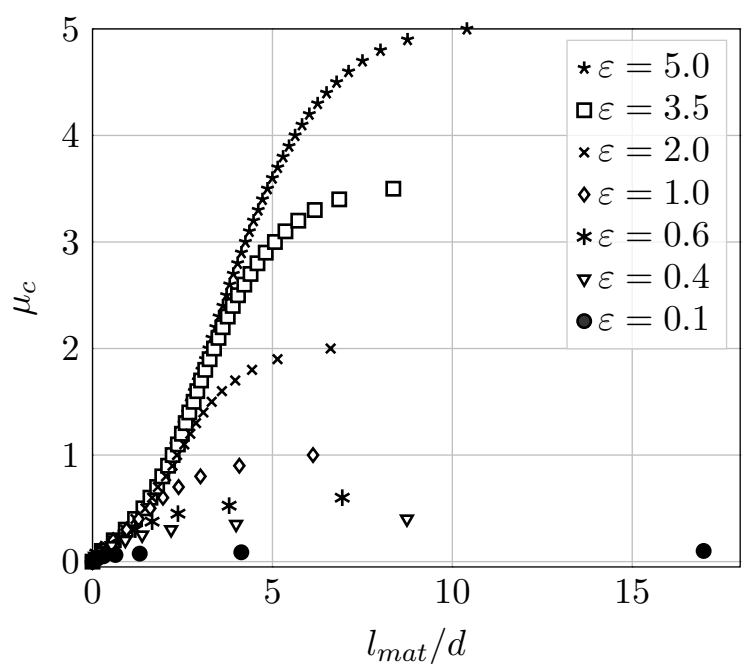

(b)

Figure 8: Dimensionless a) incremental energy release rate to square stress ratio as a function of the dimensionless crack length and b) crack length as a function of characteristic length to pore diameter ratio for several pore-to-free edge distances.

larger than $6.1 d$. Since the dimensionless stress $\tilde{\sigma}_{\varepsilon}(\mu)$ decreases down to a value smaller than 1 , if the initiation crack length is such that $\tilde{\sigma}_{\varepsilon}\left(\mu_{c}\right)<1$, it yields $T>\sigma_{c}$ crack initiation stress according to Eq. (19). Therefore, another cracking mechanisms is likely to occur such as initiation at the pore side opposite to the free edge or simultaneous initiation at both pore sides, studied in Section 5 .

\subsection{Influence of the pore-to-free edge ligament size}

In this section, we study the influence of the pore distance to the free edge on crack initiation. The material characteristic length is first set to $\ell_{\text {mat }}=1 \mathrm{~mm}$. Fig. 9 a shows the dimensionless initiation crack length $\mu_{c}$ variation as a function of the dimensionless ligament size $\varepsilon$ for several pore diameters. The corresponding initiation crack length as a function of the ligament size is also shown (Fig. 9b). The dimensionless and actual initiation crack lengths first increase with increasing (dimensionless) ligament size and then reach a plateau for ligament lengths approximately twice the material characteristic length. It means that for pore-to-free edge ligaments large enough compared to $\ell_{\text {mat }}$, the initiation crack length is independent of the ligament size. In other words, the pore is far enough from the free edge so that there is not interaction between each other. For ligament sizes smaller than $2 \ell_{\text {mat }}$, the crack initiation length increases with increasing ligament size. Fig. 10 shows the initiation remote imposed stress as a function of the ligament size for several pore diameters and two values of material strength $\left(\sigma_{c}=10 \mathrm{MPa}\right.$ or $\left.\sigma_{c}=20 \mathrm{MPa}\right)$. If the pore is located far enough from the free edge, there is no interaction between the free edge and the pore as 
mentioned previously, therefore the initiation remote imposed stress is constant and only depends on the pore diameter. However, the smaller the pore-to-free edge ligament, the smaller the remote imposed stress.

We now consider a larger material characteristic i.e. $\ell_{\text {mat }}=2 \mathrm{~mm}$. Fig. 11 shows the corresponding dimensionless initiation crack length $\mu_{c}$ variation as a function of the dimensionless ligament size $\varepsilon$ for several pore diameters. The same conclusions as before can be drawn if the pore is located far enough from the free edge for pores sufficiently large (ex: $d=1.2 \mathrm{~mm}$ ) compared to the material characteristic length. However, the dimensionless initiation crack length is close to the ligament size for sufficiently small pores (ex: $d=0.4 \mathrm{~mm}, \varepsilon \leq 2$ ). Remember that the dimensionless stress decreases from the pore to the free edge down to a value smaller than 1 . We note $\mu_{\varepsilon}^{1}$ the dimensionless crack length for which $\tilde{\sigma}_{\varepsilon}\left(\mu_{\varepsilon}^{1}\right)=1$. Therefore, if the initiation length is such that $\mu_{c}>\mu_{\varepsilon}^{1}$, crack initiation on the ligament is not likely to occur since it would require a remote imposed stress larger than $\sigma_{c}$, which is illustrated on Fig. 12, In that case, another crack initiation mechanism is expected, namely crack initiation at the pore side opposite to the free edge or simultaneous crack initiations at both pore sides.

\subsection{Configurations prohibiting ligament crack initiation}

The configurations for which crack initiation in the ligament is not likely to occur $\left(T_{c}>\sigma_{c}\right)$ are identified in this Section. They correspond to initiation crack lengths larger than $\mu_{\varepsilon}^{1}$ for which $\tilde{\sigma}_{\varepsilon}<1$. Fig. 13 a shows the dimensionless stress variation as a function of the dimensionless

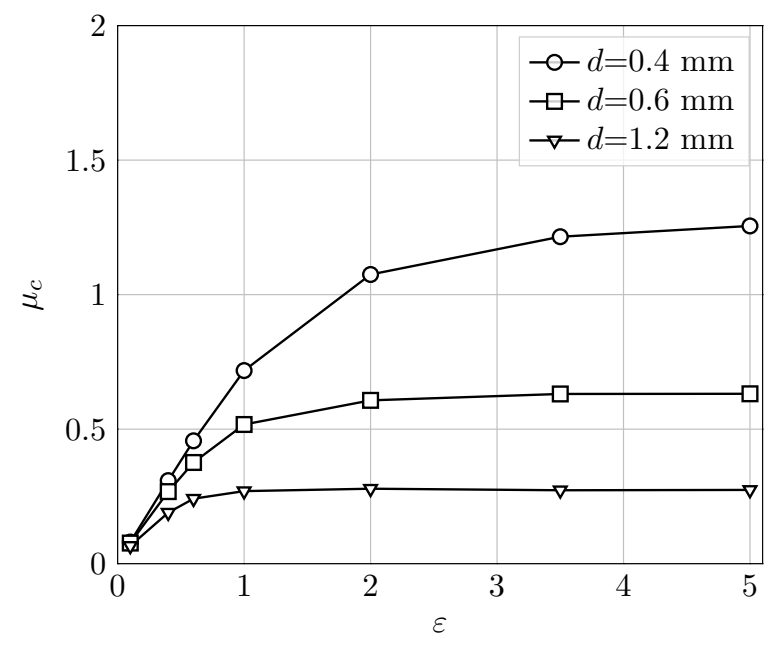

(a)

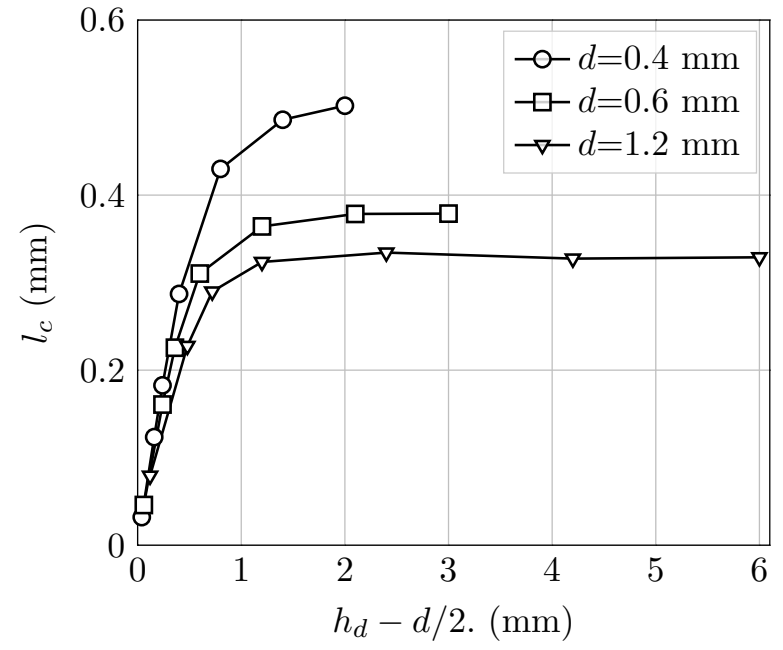

(b)

Figure 9: a) Dimensionless or b) actual initiation crack length as a function of the dimensionless pore-to-free edge ligament size for several pore diameters obtained for $\ell_{m a t}=1 \mathrm{~mm}$. 


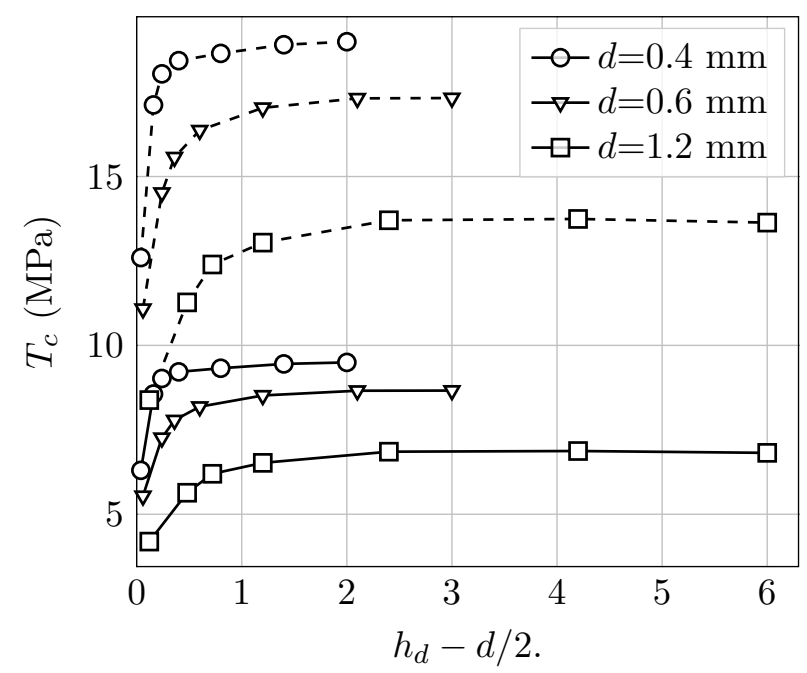

Figure 10: Initiation imposed remote stress as a function of the pore-to-free edge ligament size for several pore diameters obtained for $\ell_{m a t}=1 \mathrm{~mm}$ (dashed lines: $\sigma_{c}=20 \mathrm{MPa}$, solid lines: $\sigma_{c}=10 \mathrm{MPa}$ ).

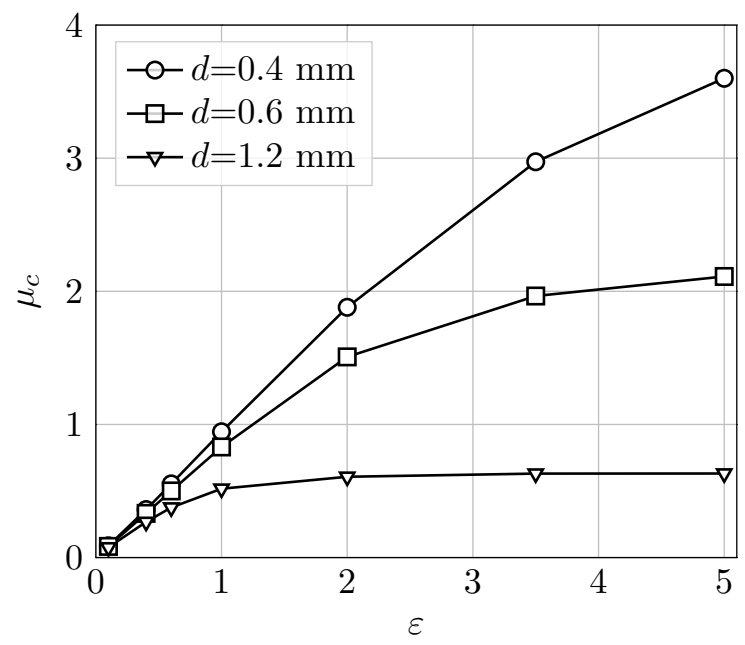

(a)

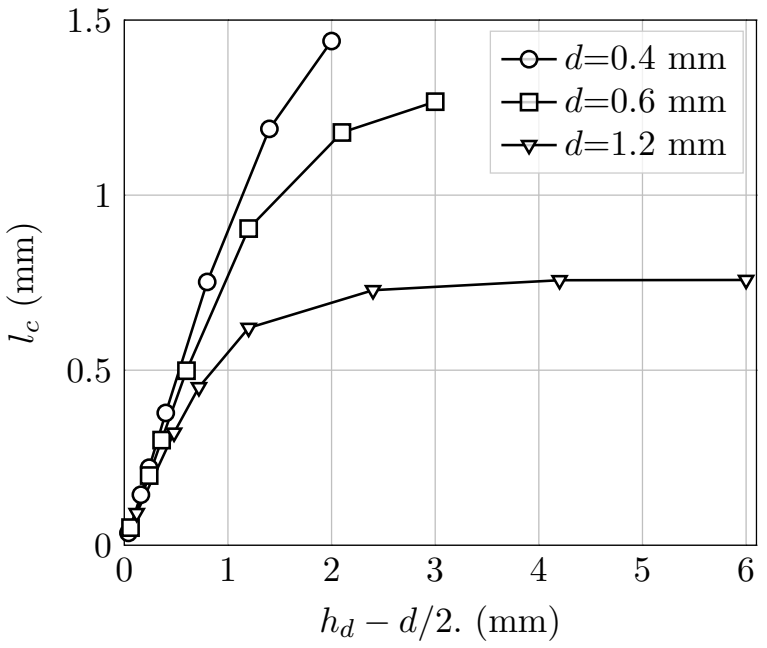

(b)

Figure 11: a) Dimensionless or b) actual initiation crack length as a function of the dimensionless pore-to-free edge ligament size for several pore diameters obtained for $\ell_{m a t}=2 \mathrm{~mm}$.

crack length for several ligament sizes and Fig. 13 b shows the variation of $\mu_{\varepsilon}^{1}$ as a function of the dimensionless ligament size. The range of dimensionless crack lengths prohibiting crack initiation in the ligament increases with increasing ligament size. Since the dimensionless initiation crack length $\mu_{c}$ depends on $\ell_{\text {mat }} / d$ (Fig. 8), it is thus possible to determine the configurations $\left(\varepsilon, \ell_{\text {mat }} / d\right.$ ) for which crack initiation in the ligament is likely to occur or not. We computed the initiation crack length and imposed remote stress as a function of $\varepsilon$ and $\ell_{\text {mat }} / d$ (Fig. 14). The configurations for which crack initiation in the ligament only are not likely to occur are determined in an equivalent manner from Fig. 14a for $\mu_{c} \geq \mu_{\varepsilon}^{1}$ or from Fig. 14p for $T / \sigma_{c} \geq 1$. 


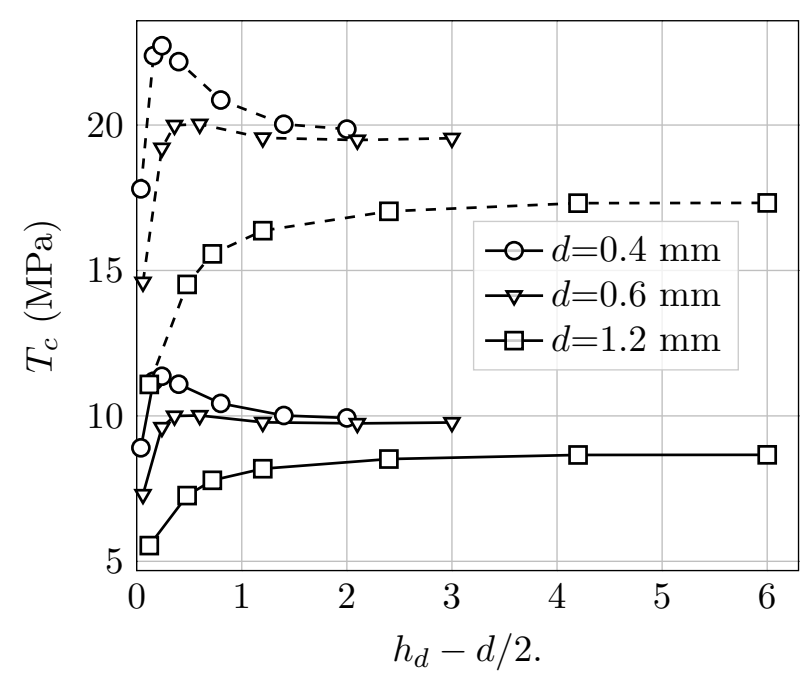

Figure 12: Initiation imposed remote stress as a function of the pore-to-free edge ligament size for several pore diameters obtained for $\ell_{\text {mat }}=2 \mathrm{~mm}$ (dashed lines: $\sigma_{c}=20 \mathrm{MPa}$, solid lines: $\sigma_{c}=10 \mathrm{MPa}$ ).

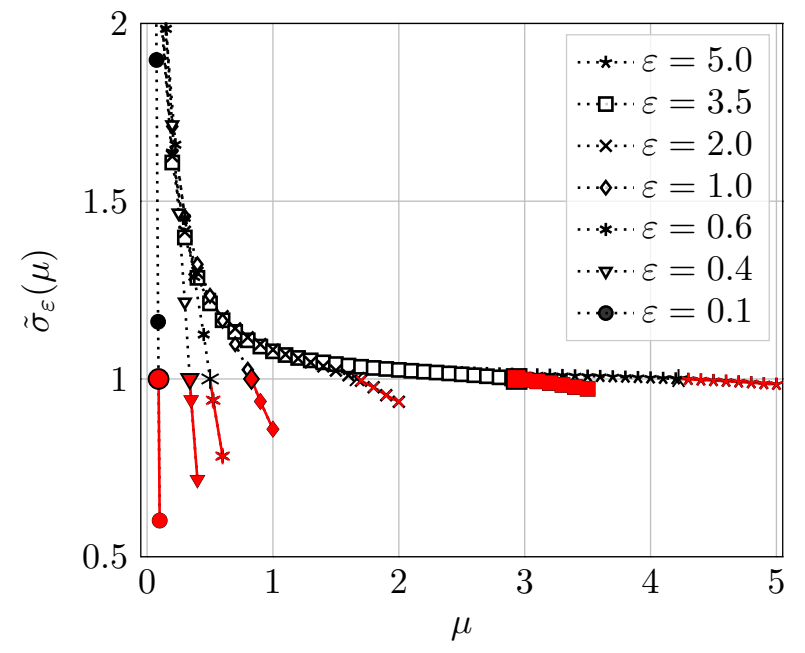

(a)

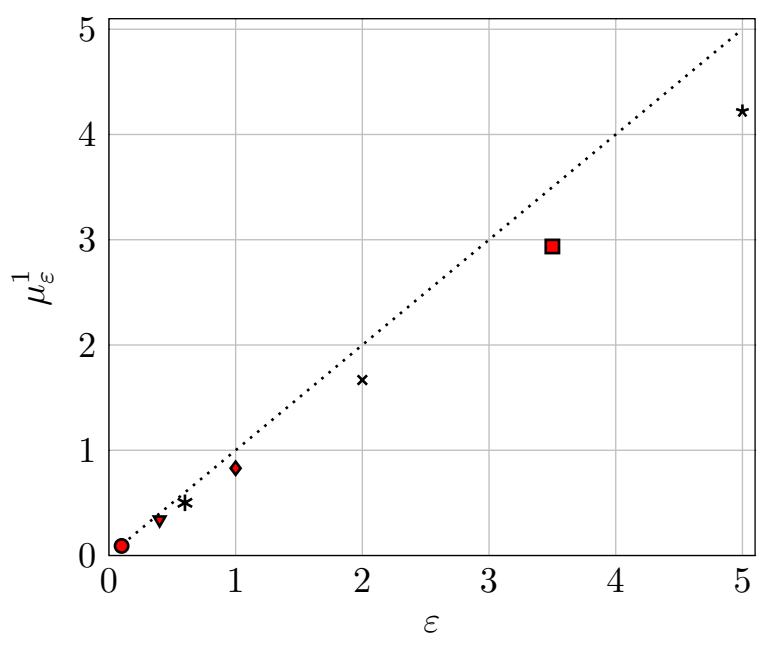

(b)

Figure 13: a) Dimensionless stress as a function of the dimensionless crack length. Colored symbols represent the ligament area for which $\tilde{\sigma}_{\varepsilon}<1, \mathrm{~b}$ ) crack length $\mu_{\varepsilon}^{1}$ corresponding to $\tilde{\sigma}_{\varepsilon}=1$ (dashed line indicates the first bisector as a guide line).

\subsection{Influence of the pore diameter}

We now assess the influence of the pore diameter on crack initiation. The material characteristic length is first set to $\ell_{\text {mat }}=0.2 \mathrm{~mm}$. Fig. 15 shows the dimensionless initiation crack length variation as a function of the pore diameter $d$ for several pore-to-free edge ligament sizes. The corresponding initiation crack length as a function of the pore diameter is also shown. The dimensionless initiation crack length decreases as a function of the pore size whatever the ligament size (Fig. 15), leading to a possible non-monotonous variation of the initiation crack length as a function of the pore size 


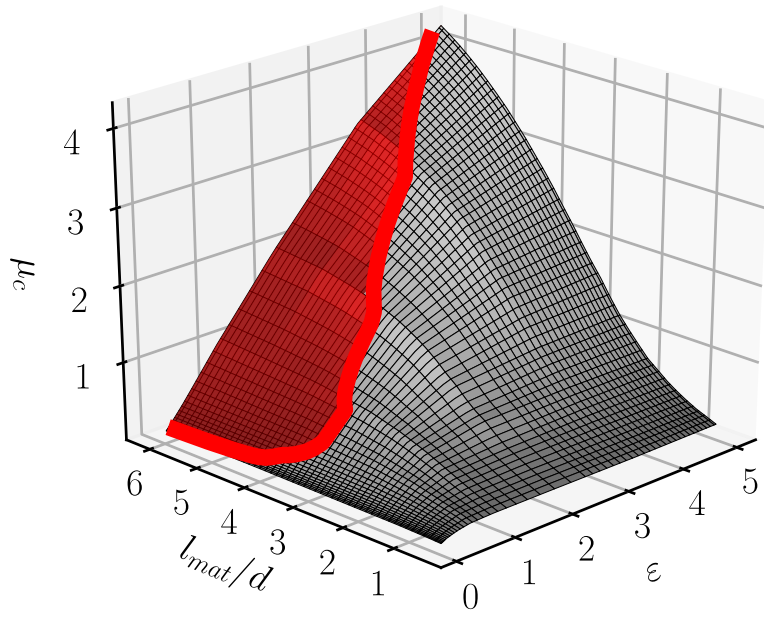

(a)

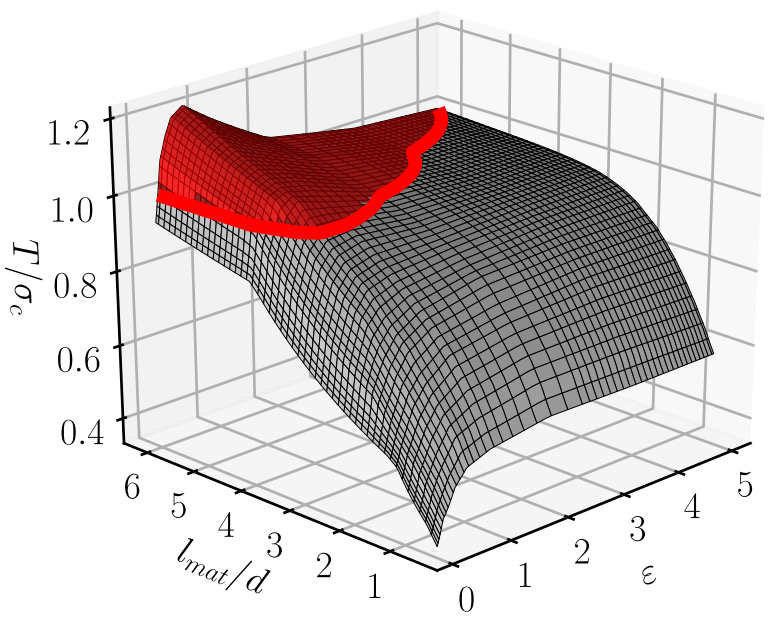

(b)

Figure 14: a) Dimensionless crack length and b) Initiation imposed remote stress to strength ratio as a function of the dimensionless pore-to-free edge ligament size and material characteristic length to pore diameter ratio. The red zones indicate the area for which $\mu_{c}>\mu_{\varepsilon}^{1}$, i.e. for which $T_{c}>\sigma_{c}$.

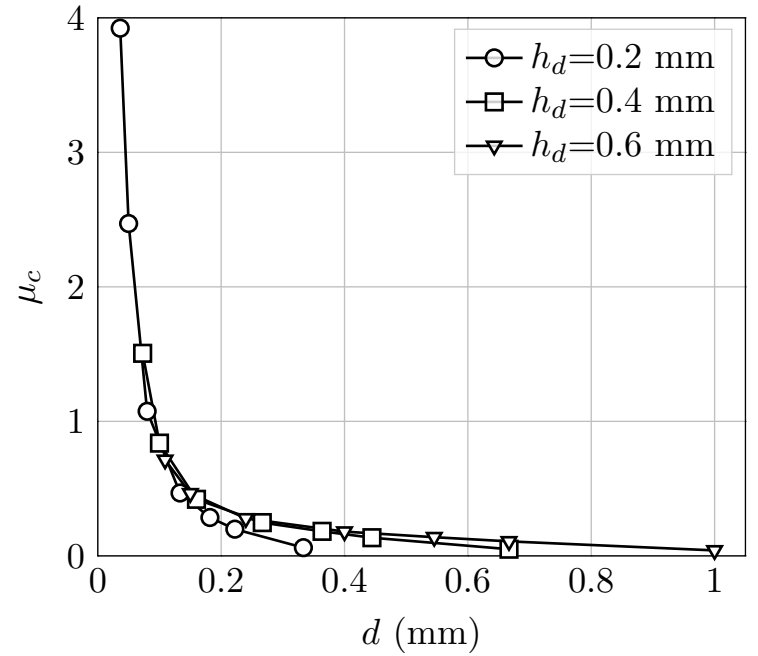

(a)

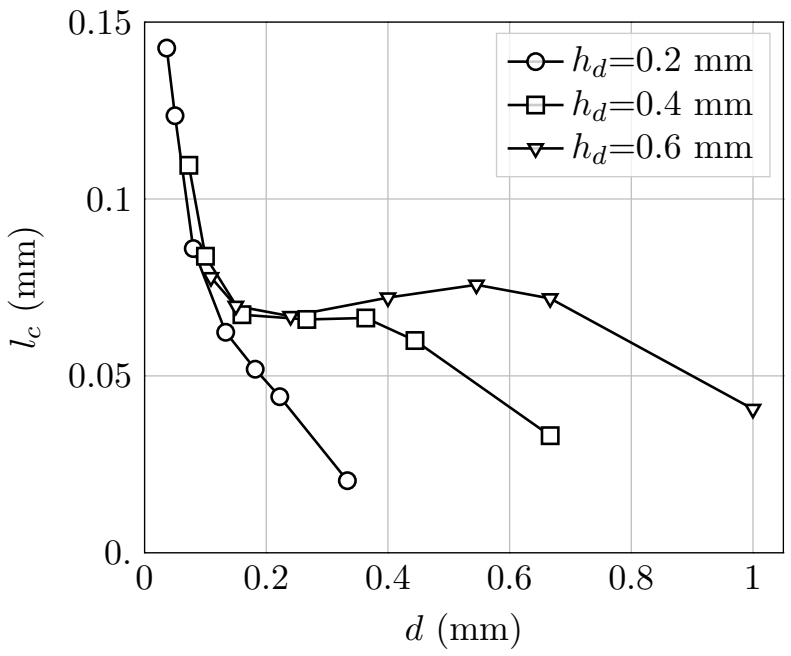

(b)

Figure 15: a) Dimensionless and b) actual initiation crack length as a function of the pore size for several pore diameters obtained for $\ell_{m a t}=0.2 \mathrm{~mm}$.

since $l_{c}=d \mu_{c}$ (Fig. 15b). Fig. 16 shows the variation of the remote imposed stress at initiation as a function of the pore size for several ligament sizes. The initiation imposed stress decreases as a function of the pore size whatever the ligament size. Morevoer, if $\ell_{m a t} \leq h_{d}$, the initiation length is smaller than $\mu_{\varepsilon}^{1}$ therefore the initiation stress does not exceed $\sigma_{c}$.

We now consider a larger material characteristic length, $\ell_{\text {mat }}=1 \mathrm{~mm}$. Fig. 17 shows the variation of the dimensionless initiation crack length $\mu_{c}$ as well as the corresponding initiation crack length $l_{c}$ variations as a function of the pore diameter $d$ for several pore-to-free edge ligament 
sizes. Both $\mu_{c}$ and $l_{c}$ are decreasing functions of the pore diameter whatever the liagement size. Since the material characteristic length is larger than the ligament size, it results in dimensionless initiation crack lengths in the range $\left[\mu_{\varepsilon}^{1}, \varepsilon\right]$ for sufficiently small pore diameters, corresponding to a configuration for which crack initiation in the ligament is not likely to occur (Fig. 18), which is all the more highlighted as the pore is close to the free edge. The initiation imposed remote stress decreases with increasing pore size whatever the pore distance to the free edge. Since the incremental energy release rate is strictly increasing within the ligament, unstable crack propagation occurs right after initiation, resulting in two possible scenarii. If the initiation stress is sufficiently high, crack re-initiation at the opposite pore edge occurs and unstable crack propagation is observed at the same remote imposed stress. Otherwise, the initiation stress must be increased to enable crack re-initiation at the opposite pore edge, as also observed in [36] in the case of adhesively bonded joint containing pores. After re-initiation, unstable crack propagation also occurs because of the monotonously increasing incremental energy release rate.

\section{Other crack initiation scenarii}

Previous sections were dedicated to predict crack initiation in the pore-to-free edge ligament. This section concerns other crack initiation mechanisms.

\subsection{Crack initiation beyond the pore}

We first study the possibility of crack initiation beyond the pore. This scenario implies that the initiation length is larger than $h_{d}+d / 2$ and that the stress criterion is fulfilled on the whole

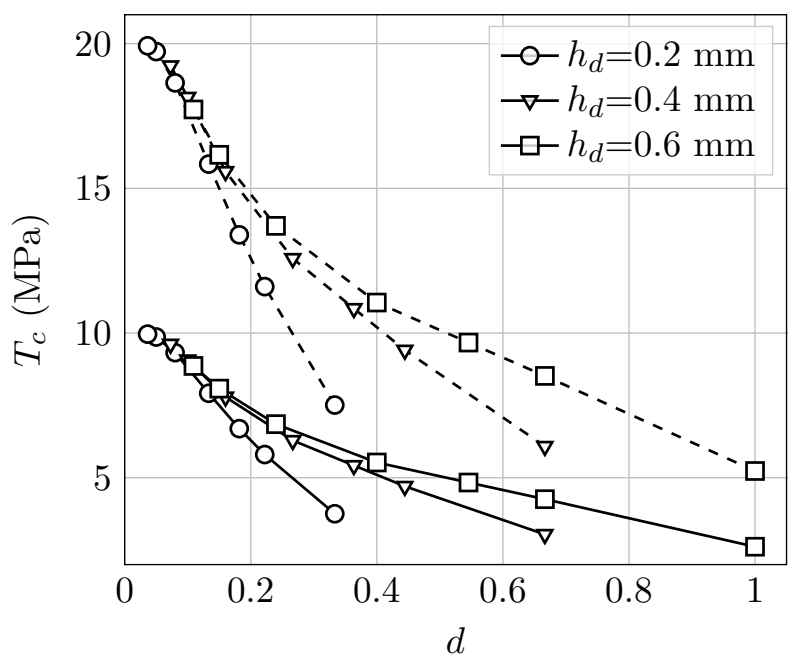

Figure 16: Initiation imposed remote stress as a function of the pore diameter for several pore-to-free edge ligament sizes obtained for $\ell_{\text {mat }}=0.2 \mathrm{~mm}$ (dashed lines: $\sigma_{c}=20 \mathrm{MPa}$, solid lines: $\sigma_{c}=10 \mathrm{MPa}$ ). 


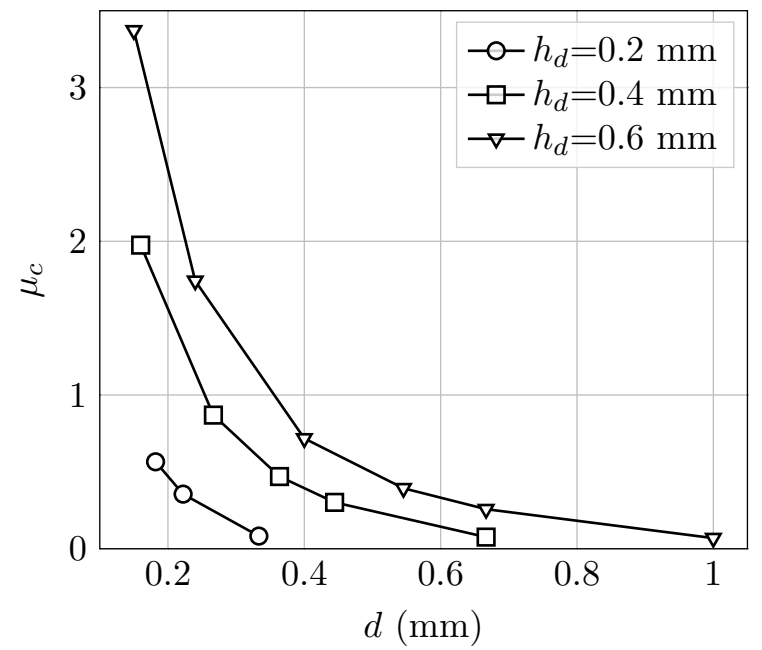

(a)

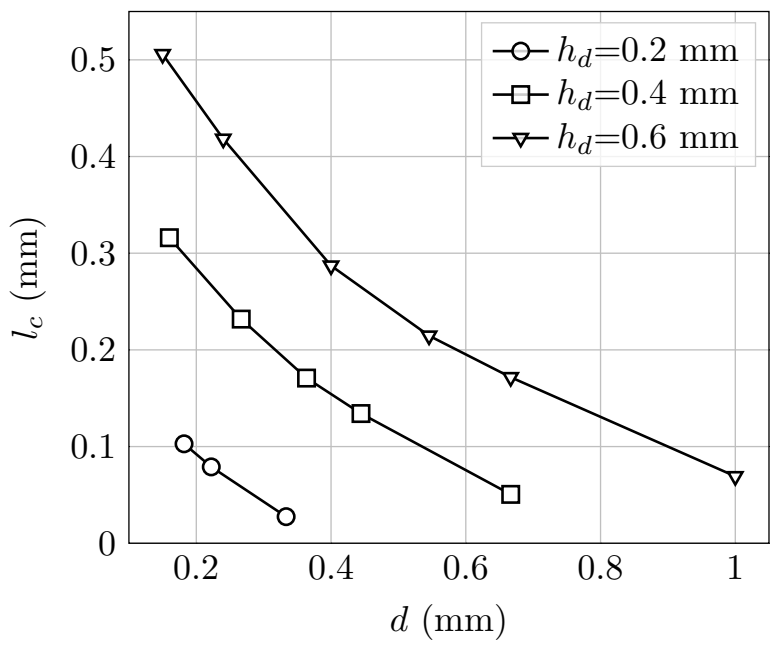

(b)

Figure 17: a) Dimensionless and b) actual initiation crack length as a function of the pore size for several pore diameters obtained for $\ell_{m a t}=1 \mathrm{~mm}$.

pore-to-free edge ligament, as well as on a certain distance starting from the pore side opposite to the free edge. Whatever the pore diameter and pore-to-free edge ligament size, the normalized stress distribution $\tilde{\sigma}_{\varepsilon}(\mu)$ on the pore side opposite to the free edge varies between around 3 close to the pore (stress concentration induced by the pore) to 1 far from the pore remote stress at infinity. Crack initiation beyond the pore is possible only if $T \tilde{\sigma}_{\varepsilon}(\mu)>\sigma_{c} \forall \mu \leq \varepsilon$. Since the stress is monotonously decreasing, this conditions reverts to $T \geq \sigma_{c} / \min \left(\tilde{\sigma}_{\varepsilon}(\mu)\right)=\sigma_{c} / \tilde{\sigma}_{\varepsilon}(\varepsilon)$. This condition also ensures that $\tilde{\sigma}_{\varepsilon}(\mu) \geq \sigma_{c} \quad \forall \mu \geq \epsilon$. Therefore, the minimum loading that allows crack initiation

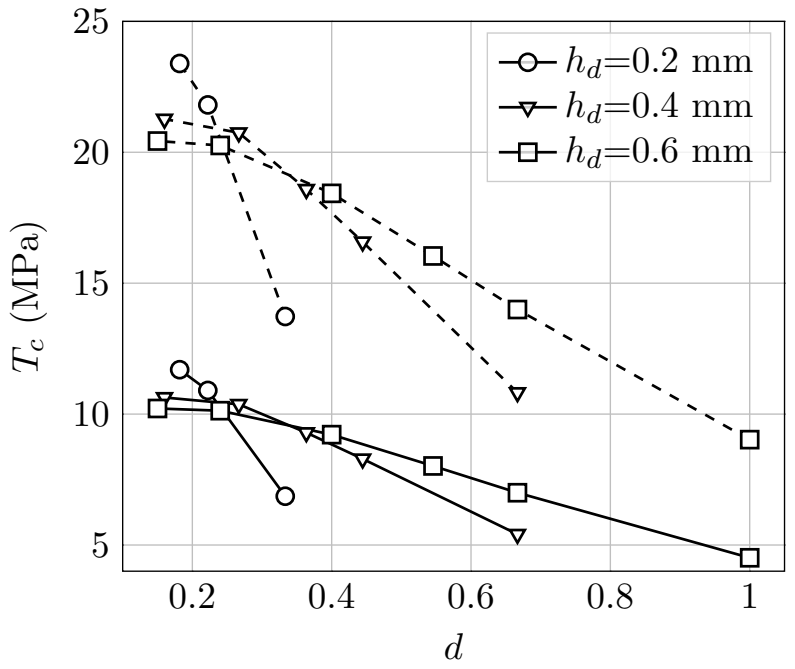

Figure 18: Initiation imposed remote stress as a function of the pore diameter for several pore-to-free edge ligament sizes obtained for $\ell_{\text {mat }}=1 \mathrm{~mm}$ (dashed lines: $\sigma_{c}=20 \mathrm{MPa}$, solid lines: $\sigma_{c}=10 \mathrm{MPa}$ ). 
beyond the pore is

$$
T=\sigma_{c} / \tilde{\sigma}_{\varepsilon}(\varepsilon)
$$

However, the energy condition must also be fulfilled to ensure crack initiation. It means that the energy condition in Eqn. (16) must hold true for at least one value of $\mu$. Combining Eqn. (16) and (23) yields the following condition:

$$
\frac{g_{\varepsilon}(\mu)}{\tilde{\sigma}_{\varepsilon}(\varepsilon)^{2}}=\frac{\ell_{m a t}}{d}
$$

The existence of a dimensionless crack length fulfilling the energy condition is actually checked since $g_{\varepsilon}$ is increasing for sufficiently large $\mu$ values so that it reaches any $\ell_{\text {mat }} / d$ for a large enough $\mu$ (Fig 19)a. We finally consider the example of a dimensionless pore-to-free edge ligament size $\varepsilon=2$ for

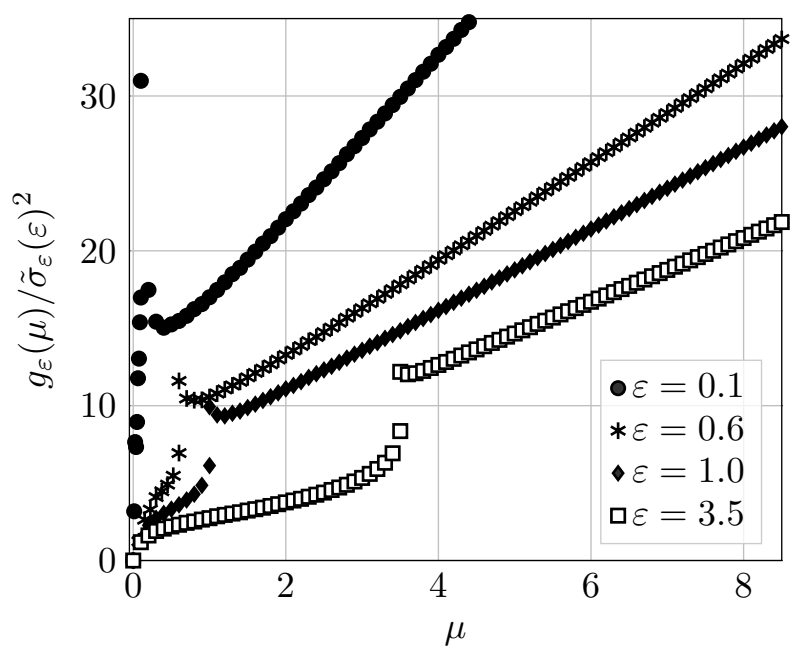

(a)

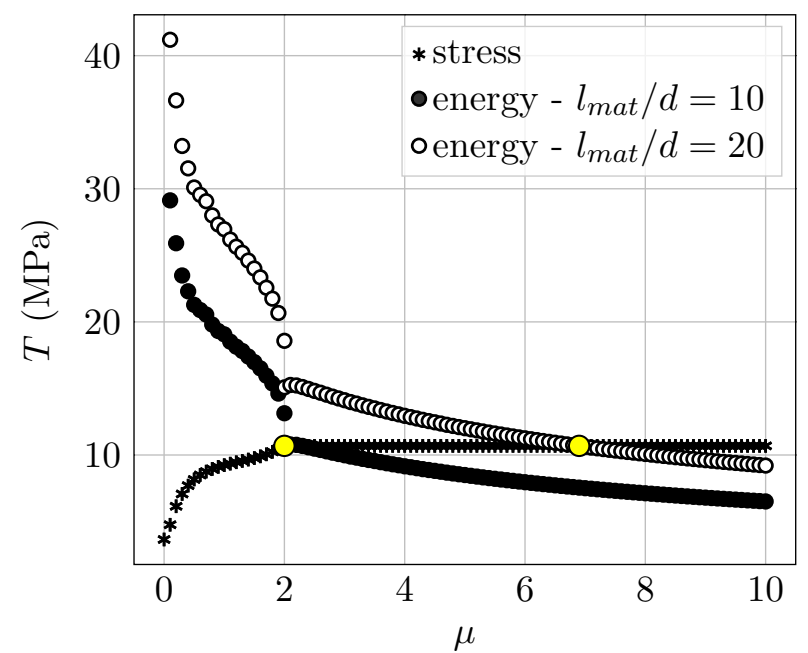

(b)

Figure 19: a) Normalized dimensionless incremental energy release rate for several dimensionless pore-to-free edge sizes and b) initiation remote imposed stress for different $\ell_{\text {mat }} / d$ as a function of dimensionless crack length highlighting a crack initiation beyond the pore.

$\ell_{\text {mat }}=10$ or $\ell_{\text {mat }}=20$. In both case, there is no solution for Eqn. 18 in the ligament. Fig. $19 \mathrm{~b}$ shows the variation of the remote stress that must be imposed in order to fulfill either the stress or the energy criteria. The dimensionless crack initiation length can be determined as the minimum loading for which both criteria are fulfilled. As explained previously, the remote stress that must be imposed to fulfill the stress criterion on a length larger than the ligament size is limited by the stress required to fulfill it on the ligament $\left(\sigma_{c} / \tilde{\sigma}_{\varepsilon}(\varepsilon)\right)$, which explains why the remote imposed stress variation presents a plateau for lengths larger than the ligament size. The dimensionless initiation crack length can be determined as the one fulfilling both criteria, it increases with increasing $\ell_{\text {mat }} / d$. 


\subsection{Crack initiation at the pore side opposite to the free edge}

The pore actually induces stress concentrations at both sides, therefore the side opposite to the free edge is also a possible crack initiation location. We thus study two additional crack initiation scenarii, namely crack initiation at the pore side opposite to the free edge and simultaneous crack initiation at both pore sides. When considering crack initiation at both pore sides, a possibility consists in assuming a symmetrical configuration with the same crack length at both pore sides [37]. However, given the asymmetry of the studied configuration, we define the potential cracks based on the stress isocontours. It means that the crack does not have the same length on both hole sides. The main advantage of this approach is that for a given crack length, the stress criterion is strictly fulfilled on the whole crack path prior to initiation. We computed the dimensionless incremental energy release rate and stress corresponding to these two scenarii for different dimensionless ligament sizes (Figs. 20 and 21). For dimensionless ligament size $\varepsilon<1$, the pore induced stress concentration

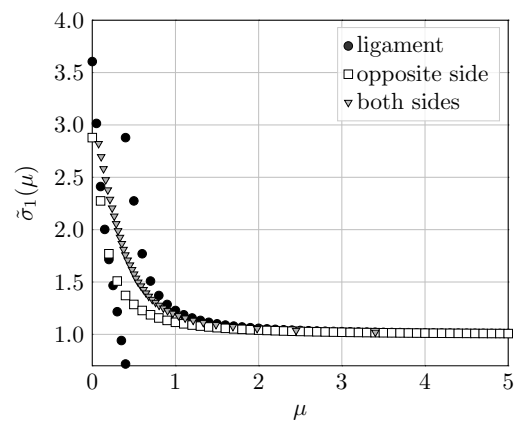

(a)

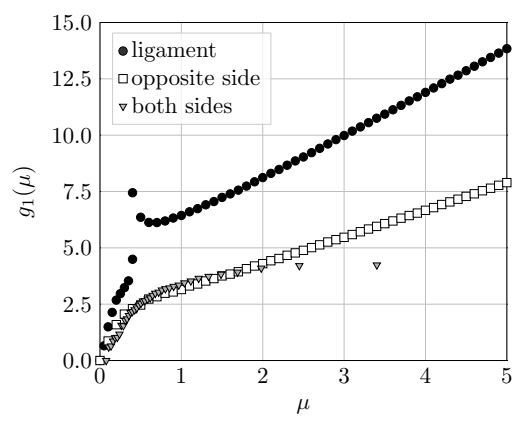

(b)

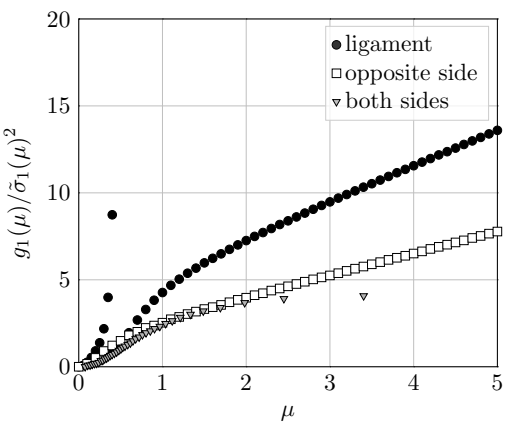

(c)

Figure 20: Dimensionless a) stress, b) incremental energy release rate and c) ratio involved in Eq. (18) as a function of dimensionless crack length for $\varepsilon=0.4$.

magnitude differs on both sides of the pore. It is larger on the ligament than on the opposite side, its magnitude increases with decreasing ligament size, see Fig. 7. The stress variation as a function of the crack length along the ligament also differs since for sufficiently small crack lengths, it is larger in the ligament than on the opposite side and conversely it is smaller in the ligament than on the opposite side for larger crack lengths (Fig. 20a). The dimensionless incremental energy release rate is larger on the ligament than on the opposite side (Fig. 20b). Qualitatively, it can thus be expected that for sufficiently small initiation crack length (or equivalently for sufficiently small $\ell_{\text {mat }} / d$, crack initiation in the ligament occurs since both the dimensionless stress and incremental energy release rate are larger than those corresponding to crack initiation at the opposite side. For larger $\ell_{\text {mat }} / d$ (or larger initiation crack lengths), however, the dimensionless stress becomes larger on the opposite side than on the ligament whereas the incremental release rate remains smaller. 
Therefore, it is not straightforward to determine which mechanism is the most likely to occur. It can only be concluded that the resulting initiation crack length is larger for crack initiation on the opposite side than on the ligament (Fig. 20k) and can be determined by solving Eqn. (18). The mechanisms that is the most likely to occur is the one corresponding to the smallest imposed remote stress. It can be noted that if two mechanisms lead to the same imposed remote initiation stress $T_{c}$, the mechanisms that is the most likely to occur is the one corresponding to the larger initiation crack length. Indeed, since the dimensionless incremental energy release rate increases with increasing dimensionless crack length, a larger crack length corresponds to a larger dissipated potential energy.

For dimensionless ligament size $\varepsilon>1$ (see Fig. 21), the pore induced stress concentration mag-

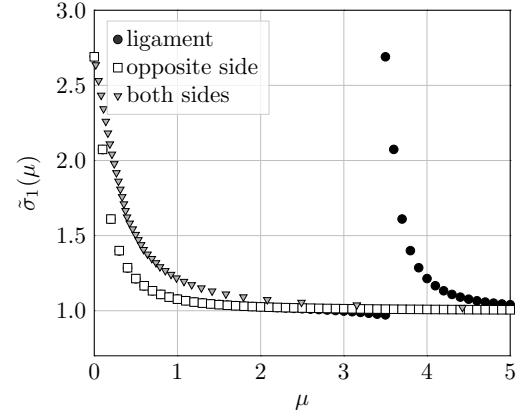

(a)

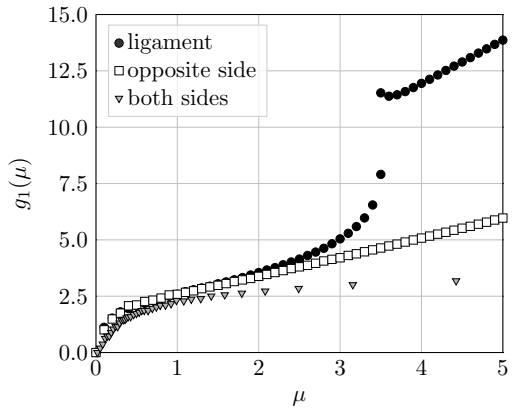

(b)

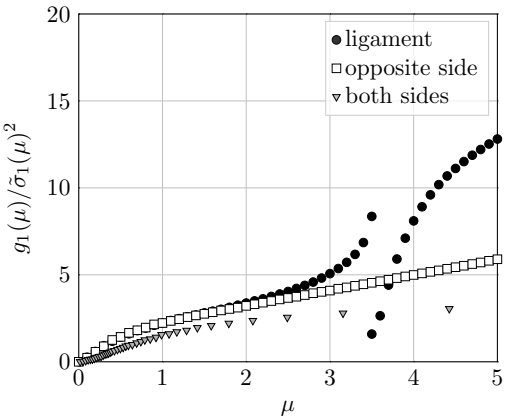

(c)

Figure 21: Dimensionless a) stress, b) incremental energy release rate and c) ratio involved in Eq. (18) as a function of dimensionless crack length for $\varepsilon=3.5$.

nitude is similar at both pore sides and the stress variation on each side of the pore is similar (curves are almost superimposed in Fig. 21a) except for crack lengths close the the ligament length $\left(\mu>\mu_{\varepsilon}^{1}\right)$ for which the dimensionless stress becomes smaller than 1 on the ligament and decreases towards 1 on the side opposite to the free edge. The dimensionless incremental energy release rate is similar on the ligament and on the opposite side for crack lengths sufficiently small with respect to the ligament size $(\mu<\varepsilon / 2)$. For larger crack lengths, it is larger on the ligament than on the opposite side. Therefore, crack initiation may occur either at the ligament or at the opposite side for initiation crack lengths sufficiently small with respect to the ligament length (or equivalently for sufficiently small $\ell_{\text {mat }} / d$. Crack initiation at the pore side opposite to the free edge is more likely for sufficiently large $\ell_{\text {mat }} / d$ so that $\mu>\mu_{\varepsilon}^{1}$ ). Whatever the dimensionless ligament size, it is not straightforward to compare either one-side initiation or simultaneous crack initiation at both sides based only on the dimensionless stress and incremental energy release rate variations. It can only be concluded from Figs. 20k and 21k that the total initiation length is larger for simultaneous 
crack initiation at both sides than for one-side crack initiation. Once again, the determination of the crack initiation configuration corresponds to the minimum initiation imposed remote stress. The dimensionless initiation crack length and normalized remote imposed stress as a function of the ligament size corresponding to one-side or simultaneous crack initiation are shown in Fig. 22 $\left(\ell_{\text {mat }} / d=0.6\right)$ and Fig. $23\left(\ell_{\text {mat }} / d=4.0\right)$. For sufficiently small $\ell_{\text {mat }} / d$ (Fig. 22$)$, the dimensionless

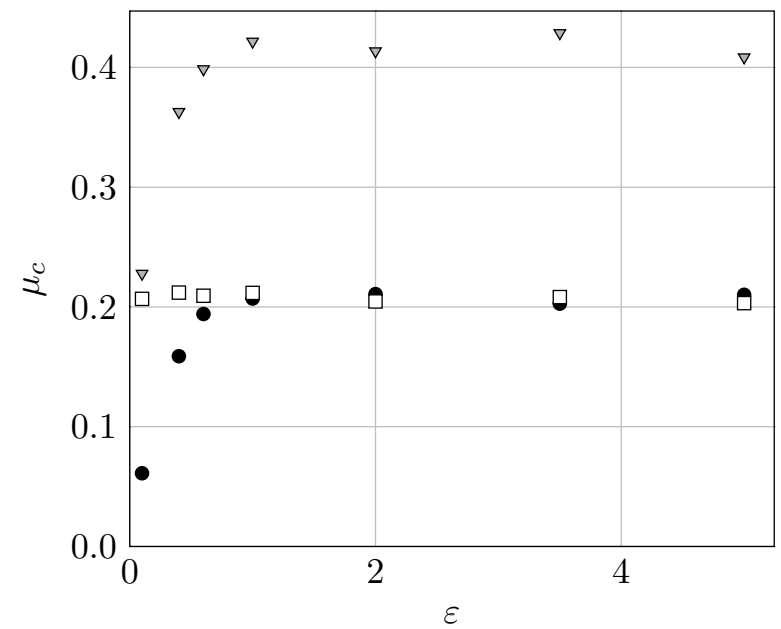

(a)

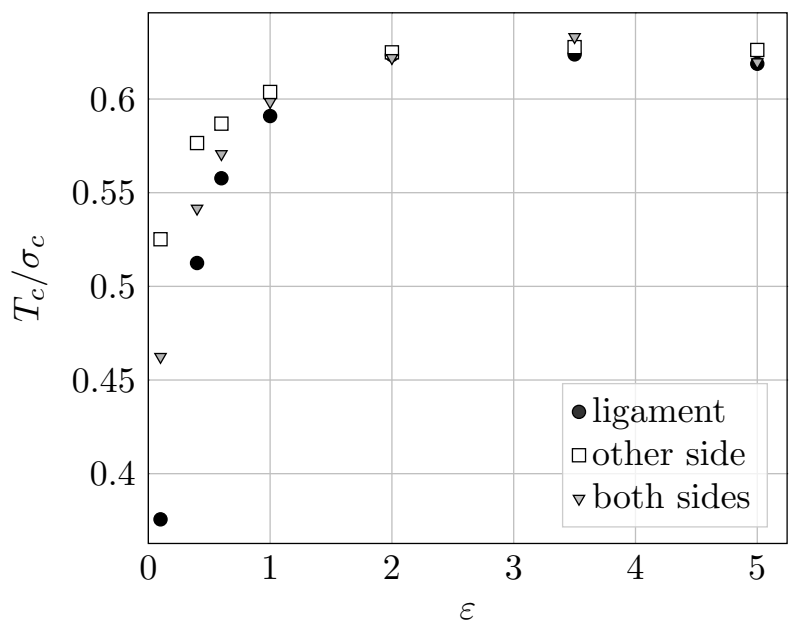

(b)

Figure 22: Initiation a) crack length and b) imposed remote stress to strength ratio as a function of the dimensionless ligament size for either crack initiation in the ligament, at the opposite pore side or simultaneously at both sides for $\ell_{\text {mat }} / d=0.6$

initiation crack length corresponding to crack initiation on the pore side opposite to the free edge remains almost constant whatever the dimensionless ligament size. The initiation imposed remote stress increases with increasing ligament size until a plateau that only depending on the pore size. For dimensionless ligament size corresponding to the plateau, it is difficult to distinguish which mechanism is the most likely to occur since they all result in similar initiation remote stress values. The maximum energy dissipation principle can be invoked to conclude that crack initiation at both sides is more likely to occur in that case since it corresponds to a larger initiation crack length and thus a larger dissipated energy. However, for dimensionless ligament size smaller than that corresponding to the plateau, crack initiation in the ligament is clearly more likely to occur since it corresponds to the smallest initiation remote imposed stress.

For larger $\ell_{m a t} / d$ (Fig. 23), the dimensionless initiation crack length increases with increasing dimensionless ligament size for the three initiation configurations. As expected, the configurations of crack initiation in the ligament leading to a remote imposed stress larger than $\sigma_{c}$ is not the most likely to occur since a smaller initiation stress is obtained for crack initiation at the pore side 
opposite to the free edge. For large enough dimensionless ligament size, both configurations lead to similar initiation remote imposed stress thus simultaneous crack initiation at both pore sides is privileged. Finally, it seems that crack initiation occurs in the ligament except for configuration

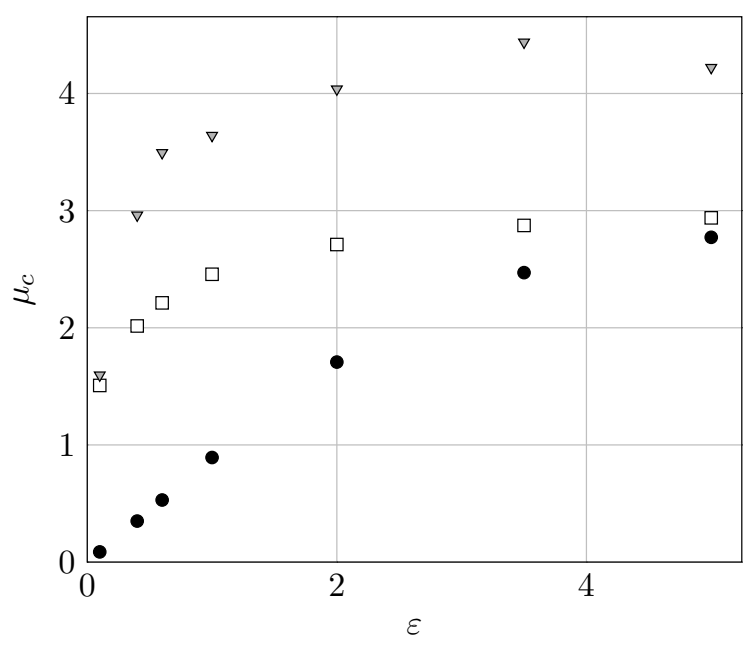

(a)

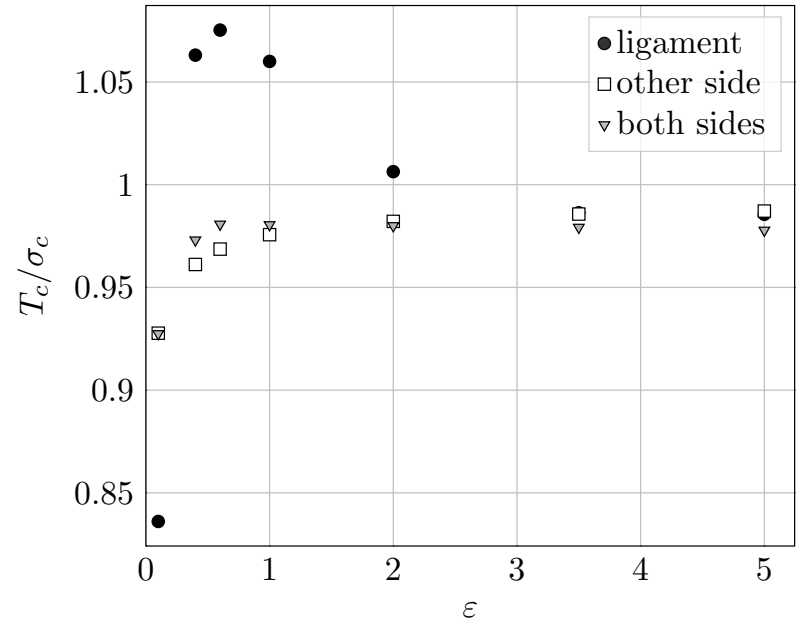

(b)

Figure 23: Initiation a) crack length and b) imposed remote stress to strength ratio as a function of the dimensionless ligament size for either crack initiation in the ligament, at the opposite pore side or simultaneously at both sides for $\ell_{\text {mat }} / d=4.0$

corresponding to the red zone depicted in Fig. 14, for which either crack initiation at the pore side opposite to the free edge (for large $\varepsilon$ ) or simultaneous crack initiation (for intermediate $\epsilon$ and large $\ell_{\text {mat }} /$ dratios) is more likely to occur. If the crack initiates on the pore side opposite to the free edge, this is expected to trigger the ligament failure because, once a crack has initiated toward the interior, it is more or less as if the pore was bigger. It is checked since it can be shown that at crack initiation $\mathcal{G} \geq G_{c}$ and that the differential energy release $\mathcal{G}$ rate increases with increasing crack length, thus resulting in unstable crack propagation right after initiation.

\section{Conclusion}

The pore to free edge ligament size strongly influences crack initiation at a pore close to a free edge. There is a competition between three possible mechanisms, namely crack initiation in the ligament, at the pore side opposite to the free edge or simultaneously at both pore sides. The mechanism that is the most likely to occur depends on two quantities: the ligament size and the material characteristic length to pore diameter ratio. If the ligament is sufficiently large, simultaneous crack initiation at both pore sides occurs, the imposed remote stress increasing up to the material tensile strength for increasing $l_{\text {mat }} / d$ ratios. For small $l_{\text {mat }} / d$ ratios and ligament 
size, crack initiation in the ligament is privileged. However, for larger $l_{\text {mat }} / d$ and small enough ligament size, crack initiation at the pore side opposite to the free edge occurs. The experimental validation of the different crack initiation scenarios is not straightforward because of unstable crack propagation occurring after initiation, contrary to configurations leading to an arrest length [38].

\section{References}

[1] Z. Hashin, Finite thermoelastic fracture criterion with application to laminate cracking analysis, J. Mech. Phys. Solids 44 (1996) 1129-1145. doi:https://doi.org/10.1016/0022-5096(95) 00080-1.

[2] J. Nairn, Exact and variational theorems for fracture mechanics of composites with residual stresses, traction-loaded cracks, and imperfect interfaces, Int. J. Fract. 105 (2000) 243-271.

[3] D. Taylor, P. Cornetti, N. Pugno, The fracture mechanics of finite crack extension, Eng. Fract. Mech. 72 (2005) 1021-1038. doi:https://doi.org/10.1016/j.engfracmech.2004.07.001.

[4] A. A. Griffith, The phenomena of rupture and flow in solids, Philosophical Transactions of the Royal Society of London A: Mathematical, Physical and Engineering Sciences 221 (582-593) (1921) 163-198.

[5] D. Leguillon, Strength or toughness? a criterion for crack onset at a notch, Eur. J. Mech. A/Solids 21(1) (2002) 61-72.

[6] P. Weißgraeber, D. Leguillon, W. Becker, A review of finite fracture mechanics: crack initiation at singular and non-singular stress raisers, Archive Appl. Mech. 86(1-2) (2016) 375-401.

[7] P. Cornetti, N. Pugno, A. Carpinteri, D. Taylor, Finite fracture mechanics: A coupled stress and energy failure criterion, Engng. Fract. Mech. 73 (2006) 2021-2033.

[8] A. Leite, V. Mantič, F. Paris, Crack onset in stretched open hole pmma plates considering linear and non-linear elastic behaviours, Theoretical and Applied Fracture Mechanics 114 (2021) 102931.

[9] P. L. Rosendahl, Y. Staudt, A. P. Schneider, J. Schneider, W. Becker, Nonlinear elastic finite fracture mechanics: modeling mixed-mode crack nucleation in structural glazing silicone sealants, Materials and Design 182 (2019) 108057. 
[10] J. Li, D. Leguillon, E. Martin, X. Zhang, Numerical implementation of the coupled criterion for damaged materials, Int. J. Sol. A/Struct 165 (2019) 93-103.

[11] A. Doitrand, A. Sapora, Nonlinear implementation of Finite Fracture Mecahnics: A case study on notched Brazilian disk samples, Int. J. Non-Linear Mech. 119 (2020) 103245.

[12] A. Doitrand, R. Estevez, D. Leguillon, Comparison between cohesive zone and coupled criterion modeling of crack initiation in rhombus hole specimens under quasi-static compression, Theor. App. Fract. Mech 99 (2019) 51-59.

[13] L. Ricardo, D. Leguillon, G. Parry, A. Doitrand, Modeling the thermal shock induced cracking in ceramics, J. Eur. Cer. Soc. 40 (2020) 1513-1521.

[14] D. Leguillon, E. Sanchez-Palencia, Computation of Singular Solutions in Elliptic Problems and Elasticity, Wiley, USA, 1987.

[15] Z. Yosibash, E. Priel, D. Leguillon, A failure criterion for brittle elastic materials under mixed-mode loading, Int. J. Fract. 141 (2006) 291-312. doi:https://doi.org/10.1007/ s10704-006-0083-6.

[16] E. Priel, Z. Yosibash, D. Leguillon, Failure initiation at a blunt v-notch tip under mixed mode loading, Int. J.Fract 149 (2008) 142-173. doi:https://doi.org/10.1007/ s10704-008-9234-2.

[17] J. Hebel, R. Dieringer, W. Becker, Modeling brittle crack formation at geometrical and material discontinuities using a finite fracture mechanics approach, Engng. Fract. Mech. 77 (2010) 35583572. doi:https://doi.org/10.1016/j.engfracmech.2010.07.005.

[18] E. Martin, T. Vandellos, D. Leguillon, N. Carrère, Initiation of edge debonding: coupled criterion versus cohesive zone model, Int. J.Fract 199 (2016) 157-168.

[19] A. Doitrand, E. Martin, D. Leguillon, Numerical implementation of the coupled criterion: Matched asymptotic and full finite element approaches, Fin. Elem. Anal. Des. 168 (2020) 103344.

[20] A. Carpinteri, P. Cornetti, A. Sapora, A finite fracture mechanics approach to the asymptotic behaviour of u-notched structures, Fatigue and Fracture of Engineering Materials and Structures 35 (5) (2012) 451-457. 
[21] A. Sapora, P. Cornetti, Crack onset and propagation stability from a circular hole under biaxial loading, Int. J. Fract. 214 (1) (2018) 97-104.

[22] P. L. Rosendahl, P. Weißgraeber, N. Stein, W. Becker, Asymmetric crack onset at open-holes under tensile and in-plane bending loading, International Journal of Solids and Structures $113-114$ (2017) 10-23.

[23] J. Li, X. Zhang, A criterion study for non-singular stress concentrations in brittle or quasibrittle materials, Engineering Fracture Mechanics 73 (2006) 505-523.

[24] A. Sapora, A. R. Torabi, S. Etesam, P. Cornetti, Finite fracture mechanics crack initiation from a circular hole, Fatigue \& Fracture of Engineering Materials \& Structures 41 (7) (2018) $1627-1636$.

[25] A. Doitrand, P. Cornetti, A. Sapora, R. Estevez, Experimental and theoretical characterization of mixed mode brittle failure from square holes, Int. J. Fract. (2021). doi:https://doi.org/ $10.1007 / \mathrm{s} 10704-020-00512-9$.

[26] R. Romani, M. Bornert, D. Leguillon, R. Le Roy, K. Sab, Detection of crack onset in double cleavage drilled specimens of plaster under compression by digital image correlation - theoretical predictions based on a coupled criterion, Eur. J. Mech. A/Sol. 51 (2015) 172-182.

[27] E. Martin, D. Leguillon, N. Carrère, A coupled strength and toughness criterion for the prediction of the open hole tensile strength of a composite plate, Int. J. Sol. Struct. 49(26) (2012) $3915-3922$.

[28] E. Martin, D. Leguillon, N. Carrère, An extension of the point-stress criterion based on a coupled stress and energy fulfilment: application to the prediction of the open-hole tensile strength of a composite plate, Woodhead Publishing, 2015, Ch. 17, pp. 425-444. doi:http: //dx.doi.org/10.1016/B978-0-08-100137-0.00017-1.

[29] P. Camanho, G. Ercin, G. Catalanotti, S. Mahdi, P. Linde, A finite fracture mechanics model for the prediction of the open-hole strength of composite laminates, Composites Part A 43 (2012) 1219-1225.

[30] G. Catalanotti, P. Camanho, A semi-analytical method to predict net-tension failure of mechanically fastened joints in composite laminates, Composites Science and Technology 76 (2013) $69-76$. 
[31] J. Felger, N. Stein, W. Becker, Mixed-mode fracture in open-hole composite plates of finitewidth: An asymptotic coupled stress and energy approach, International Journal of Solids and Structures 122-123 (2017) 14-24.

[32] D. Leguillon, D. Quesada, C. Putot, E. Martin, Size effects for crack initiation at blunt notches or cavities, Engng. Fract. Mech. 74 (2007) 2420-2436.

[33] D. Leguillon, R. Piat, Fracture of porous materials - influence of the pore size, Engineering Fracture Mechanics 75 (2008) 1840-1853.

[34] A. Doitrand, R. Henry, I. Zacharie-Aubrun, G. J.M., S. Meille, UO $\mathrm{U}_{2}$ micron scale specimen fracture : Parameter identification and influence of porosities, Theor. Appl. Fract. Mech. 108 (2020) 102665.

[35] P. Labossiere, M. Dunn, Stress intensities at interface corners in anisotropic bimaterials., Engng. Fract. Mech. 62 (1999) 555-575.

[36] N. Carrère, A. Doitrand, E. Martin, D. Leguillon, Theoretical study based on 2d assumptions of the influence of small pores on crack initiation in adhesively bonded joints, International Journal of Adhesion and Adhesives 111 (2021) 102979.

[37] M. Muñoz Reja, L. Távara, V. Mantič, P. Cornetti, A numerical implementation of the coupled criterion of finite fracture mechanics for elastic interfaces, Theor. App. Fract. Mech. (2020). doi:10.1016/j.tafmec.2020.102607.

[38] A. Doitrand, R. Estevez, D. Leguillon, Experimental characterization and numerical modeling of crack initiation in rhombus hole pmma specimens under compression, Eur. J. Mech. Sol. 76 (2019) 290-299. 